\title{
The Variable Discharge of Cortical Neurons: Implications for Connectivity, Computation, and Information Coding
}

\author{
Michael N. Shadlen ${ }^{1}$ and William T. Newsome ${ }^{2}$ \\ ${ }^{1}$ Department of Physiology and Biophysics and Regional Primate Research Center, University of Washington, Seattle, \\ Washington 98195-7290, and 2Howard Hughes Medical Institute and Department of Neurobiology, Stanford University \\ School of Medicine, Stanford, California 94305
}

\begin{abstract}
Cortical neurons exhibit tremendous variability in the number and temporal distribution of spikes in their discharge patterns. Furthermore, this variability appears to be conserved over large regions of the cerebral cortex, suggesting that it is neither reduced nor expanded from stage to stage within a processing pathway. To investigate the principles underlying such statistical homogeneity, we have analyzed a model of synaptic integration incorporating a highly simplified integrate and fire mechanism with decay. We analyzed a "high-input regime" in which neurons receive hundreds of excitatory synaptic inputs during each interspike interval. To produce a graded response in this regime, the neuron must balance excitation with inhibition. We find that a simple integrate and fire mechanism with balanced excitation and inhibition produces a highly variable interspike interval, consistent with experimental data. Detailed information about the temporal pattern of synaptic inputs cannot be recovered from the pattern of output spikes, and we infer that cortical neurons are unlikely to transmit information in the
\end{abstract}

Since the earliest single-unit recordings, it has been apparent that the irregularity of the neural discharge might limit the sensitivity of the nervous system to sensory stimuli (for review, see Rieke et al., 1997). In visual cortex, for example, repeated presentations of an identical stimulus elicit a variable number of action potentials (Schiller et al., 1976; Dean, 1981; Tolhurst et al., 1983; Vogels et al., 1989; Snowden et al., 1992; Britten et al., 1993), and the time between successive action potentials [interspike interval (ISI)] is highly irregular (Tomko and Crapper, 1974; Softky and Koch, 1993). These observations have led to numerous speculations on the nature of the neural code (Abeles, 1991; Konig et al., 1996; Rieke et al., 1997). On the one hand, the irregular timing of spikes could convey information, imparting broad information bandwidth on the neural spike train, much like a Morse code. Alternatively this irregularity may reflect noise, relegating the signal carried by the neuron to a crude estimate of spike rate.

\footnotetext{
Received Sept. 15, 1997; revised Feb. 25, 1998; accepted March 3, 1998.

This research was supported by National Institutes of Health Grants EY05603, RR00166, EY11378 and the McKnight Foundation. W.T.N. is an Investigator of the Howard Hughes Medical Institute.

We are grateful to Richard Olshen, Wyeth Bair, and Haim Sompolinsky for advice on mathematics, Marjorie Domenowske for help with illustrations, and Crista Barberini, Bruce Cumming, Greg DeAngelis, Eb Fetz, Greg Horwitz, Kevan Martin, Mark Mazurek, Jamie Nichols, and Fred Rieke for helpful suggestions. We are also grateful to two anonymous reviewers whose thoughtful remarks improved this paper considerably.

Correspondence should be addressed to Dr. Michael N. Shadlen, Department of Physiology, University of Washington Medical School, Box 357290, Seattle, WA 98195-7290.

Copyright (C) 1998 Society for Neuroscience $\quad 0270-6474 / 98 / 183870-27 \$ 05.00 / 0$
}

temporal pattern of spike discharge. Rather, we suggest that quantities are represented as rate codes in ensembles of 50100 neurons. These column-like ensembles tolerate large fractions of common synaptic input and yet covary only weakly in their spike discharge. We find that an ensemble of 100 neurons provides a reliable estimate of rate in just one interspike interval (10-50 msec). Finally, we derived an expression for the variance of the neural spike count that leads to a stable propagation of signal and noise in networks of neurons - that is, conditions that do not impose an accumulation or diminution of noise. The solution implies that single neurons perform simple algebra resembling averaging, and that more sophisticated computations arise by virtue of the anatomical convergence of novel combinations of inputs to the cortical column from external sources.

Key words: noise; rate code; temporal coding; correlation; interspike interval; spike count variance; response variability; visual cortex; synaptic integration; neural model
In principle we could ascertain which view is correct if we knew how neurons integrate synaptic inputs to produce spike output. One possibility is that specific patterns or coincidences of presynaptic events give rise to precisely timed postsynaptic spikes. Accordingly, the output spike train would reflect the precise timing of relevant presynaptic events (Abeles, 1982, 1991; Lestienne, 1996). Alternatively, synaptic input might affect the probability of a postsynaptic spike, whereas the precise timing is left to chance. Then presynaptic inputs would determine the average rate of postsynaptic discharge, but spike times, patterns, and intervals would not convey information.

In this paper we propose that the irregular ISI arises as a consequence of a specific problem that cortical neurons must solve: the problem of dynamic range or gain control. Cortical neurons receive $3000-10,000$ synaptic contacts, $85 \%$ of which are asymmetric and hence presumably excitatory (Peters, 1987; Braitenberg and Schüz, 1991). More than half of these contacts are thought to arise from neurons within a 100-200 $\mu \mathrm{m}$ radius of the target cell, reflecting the stereotypical columnar organization of neocortex. Because neurons within a cortical column typically share similar physiological properties, the conditions that excite one neuron are likely to excite a considerable fraction of its afferent input as well (Mountcastle, 1978; Peters and Sethares, 1991), creating a scenario in which saturation of the neuron's firing rate could easily occur. This problem is exacerbated by the fact that EPSPs from individual axons appear to exert substantial impact on the membrane potential (Mason et al., 1991; Otmakhov 
et al., 1993; Thomson et al., 1993b; Matsumura et al., 1996). An individual EPSP depolarizes the membrane by $3-10 \%$ of the necessary excursion from resting potential to spike threshold, and this seems to hold for synaptic contacts throughout the dendrite regardless of the distance between synapse and soma (Hoffman et al., 1997), suggesting that a large fraction of the synapses are capable of influencing somatic membrane potential. Absent inhibition, a neuron ought to produce an action potential whenever 10-40 input spikes arrive within 10-20 msec of each other.

These findings begin to reveal the full extent of the cortical neuron's problem. The neuron computes quantities from large numbers of synaptic input, yet the excitatory drive from only 10-40 inputs, discharging at an average rate of 100 spikes/sec, should cause the postsynaptic neuron to discharge near 100 spikes/sec. If as few as 100 excitatory inputs are active (of the $\geq 3000$ available), the postsynaptic neuron should discharge at a rate of $\geq 200$ spikes/sec. It is a wonder, then, that the neuron can produce any graded spike output at all. We need to understand how cortical neurons can operate in a regime in which many (e.g., $\geq 100$ ) excitatory inputs arrive for every output spike. We will refer to this as a "high-input regime" to distinguish it from situations common in subcortical structures in which the activity of a few inputs determines the response of the neuron. We emphasize that we refer only to the active inputs of a neuron, which may be as few as $5-10 \%$ of its afferent synapses, although our arguments apply to all larger fractions as well. The actual fraction active is not known for any cortical neuron, but most cortical physiologists realize that large numbers of neurons are activated by simple stimuli (McIlwain, 1990) and would probably estimate the fraction as considerably greater than $5-10 \%$.

In this paper we analyze a simple model of synaptic integration that permits presynaptic and postsynaptic neurons to respond over the same dynamic range, solving the gain control problem. The model is a variant of the random walk model proposed by Gerstein and Mandelbrot (1964) and others (for review, see Tuckwell, 1988). Although constrained by neural membrane biophysics, the model is not a biophysical implementation. There are no synaptic or voltage-gated conductances, etc. Instead, we have chosen to attack the problem of synaptic integration as a counting problem, focusing on the consequences of counting input spikes to produce output spikes. We show in Appendix 1, however, that a more realistic, conductance-based model undergoes the same statistical behavior.

The paper is divided into three main parts. The first concerns the problem of synaptic integration in the high-input regime. Given a plethora of synaptic input, how do neurons achieve an acceptable dynamic range of response? It turns out that the solution to this problem imposes a high degree of irregularity on the pattern of action potentials - the price of a reasonable dynamic range is noise. The rest of the paper concerns implications of this noise on the reliability of neural signals. Part 2 explores the consequences of shared connections among neurons. Redundancy is a natural strategy for encoding information in noisy neurons and is a well established principle of cortical organization (Mountcastle, 1957). We examine its implications for correlation, synchrony, and coding fidelity. In part 3 we consider how neurons can receive variable inputs, compute with them, and produce a response with variability that is, on average, neither greater nor less than its inputs. We find a stable solution to the propagation of noise in networks of neurons and in so doing gain insight into the nature of neural computation itself. Together the exercise supports a view of neuronal coding and computation that requires large numbers of connections, much redundancy, and, consequentially, a great deal of noise.

\section{BACKGROUND: THE OBSERVED VARIABILITY OF SINGLE NEURONS}

The variability of the neuronal response is characterized in two ways: interval statistics and count statistics. Interval statistics refer to the time between successive action potentials, known as the ISI. For cortical neurons, the ISI is highly irregular. Because this interval is simply the reciprocal of the discharge rate at any instant, a neuron that modulates its discharge rate over time must exhibit variability in its ISIs. Yet even a neuron that fires at a constant rate over some epoch will exhibit considerable variability among its ISIs. In fact the distribution of ISIs resembles the exponential probability density of a random (Poisson) point process. To a first approximation, the time to the next spike depends only on the expected rate and is otherwise random.

Count statistics refer to the number of spikes produced in an epoch of fixed duration. Under experimental conditions it is possible to estimate the mean and variability of the spike count by repeating the measurement many times. A typical example is the number of spikes produced by a neuron in the primary visual cortex when a bar of light is passed through its receptive field. For cortical neurons, repeated presentations of the identical stimulus yield highly variable spike counts. The variance of spike counts over repeated trials has been measured in several visual cortical areas in monkey and cat. The relationship between the count variance and the count mean is linear when plotted on log-log graph, with slope just greater than unity. A reasonable approximation is that the response variance is about 1.5 times the mean response (Dean, 1981; Tolhurst et al., 1983; Bradley et al., 1987; Scobey and Gabor, 1989; Vogels et al., 1989; Snowden et al., 1992; Britten et al., 1993; Softky and Koch, 1993; Geisler and Albrecht, 1997).

What is particularly striking about both interval and counting statistics is that they seem to be fairly homogeneous throughout the cerebral cortex (Softky and Koch, 1993; Lee et al., 1998). Measurements of ISI variability are difficult, because any measured variation is only meaningful if the rate is a constant. Nevertheless, the sound of a neural spike train played through a loudspeaker is remarkably similar in most regions of the neocortex and contrasts remarkably with subcortical spike trains, whose regularity often evokes tones. Such gross homogeneity among cortical areas implies that the inputs to, and the outputs from, a typical cortical neuron conform to common statistical principles. To the electrophysiologist recording from neurons in cortical columns, it is clear that nearby neurons respond under similar conditions and that their response magnitudes are roughly similar. Neurons encountered within the column are fairly representative of the inputs of any one neuron and, in a rough sense, the targets of any one neuron (Braitenberg and Schüz, 1991). Again, we emphasize that it is only the neuron's active inputs to which we refer.

Table 1 lists properties of the neural response that apply more or less equivalently to a neuron as well as to its inputs and its targets. These properties are to be interpreted as rough rules of thumb, but they pose important constraints for the flow of impulses and information through networks of cortical neurons.

Figure 1 illustrates these properties for a neuron recorded from the middle temporal visual area (MT or V5) of a behaving monkey, a visual area that is specialized for processing motion information (for review, see Albright, 1993). Figure 1 shows 210 


\begin{tabular}{ll}
\hline Table 1. Properties of statistical homogeneity for cortical neurons \\
Response property & Approximate value \\
\hline $\begin{array}{l}\text { Dynamic range of response } \\
\text { Distribution of interspike }\end{array}$ & $0-200$ spikes/sec \\
intervals & Approximately exponential \\
Spike count variance & Variance $\sim 1-1.5$ times the mean \\
& count \\
Spike rate modulation & Expected rate can vary in $\sim 1$ ISI, \\
& $5-10$ msec \\
\hline
\end{tabular}

repetitions of the spike train produced by this neuron when an identical sequence of random dots was displayed dynamically in the receptive field of the neuron. The stimulus contained rapid fluctuations of luminance and random motion, which produced similarly rapid fluctuations in the neural discharge. The fluctuations in discharge appear stereotyped from trial to trial, as is evident from the vertical stripe-like structure in the raster and from the peristimulus time histogram (PSTH) below. The PSTH shows the average response rate calculated in $2 \mathrm{msec}$ epochs. The spike rate varied between 15 and 220 impulses/sec (mean $\pm 2 \sigma)$. A power spectral density analysis of this rate function reveals significant modulation at $50 \mathrm{~Hz}$, suggesting that the neuron is capable of expressing a change in its rate of discharge every 10 msec or less (Bair and Koch, 1996). Thus the neuron is capable of computing quantities over an interval comparable to the average ISI of an active neuron.

At first glance, the pattern of spike arrival times appears fairly consistent from trial to trial, but this turns out to be illusory. A closer examination of any epoch reveals considerable variability in both the time of spikes and their counts. Figure $1 B$ magnifies the spikes occurring between 360 and $460 \mathrm{msec}$ after stimulus onset for 50 consecutive trials, corresponding to the shaded region of Figure $1 A$. We selected this subset of the raster, because the discharge rate was fairly constant during this epoch and because it represents one of the more consistent patterns of spiking in the record. Nevertheless, the ISIs show marked variability. The mean is $7.35 \mathrm{msec}$, and the SD is 5.28 . We will frequently refer to the ratio, $\mathrm{SD} /$ mean, as the coefficient of variation of the ISI distribution $\left(C_{\mathrm{V}_{\mathrm{ISI}}}\right)$. The value from these intervals is 0.72 . The ISI frequency histogram (Fig. $1 C$ ) is fit reasonably well by an exponential distribution (solid curve)-the expected distribution of interarrival times for a random (Poisson) point process. Although some of the variability in the ISIs may be attributable to fluctuations in spike rate, the pattern of spikes is clearly not the same from trial to trial.

This point is emphasized further by simply counting the spikes produced during the epoch. If the pattern of spikes were at all reproducible, we would expect consistency in the spike count. The mean for the highlighted epoch was 12.8 spikes, and its variance was 8.22 . We made similar calculations of the mean count and its associated variance for randomly selected epochs lasting from 100 to $500 \mathrm{msec}$, including from 50 to 200 consecutive trials. The mean and variance for 500 randomly chosen epochs are shown by the scatter plot in Figure $1 D$. The main diagonal in this graph, $($ Var $=$ mean $)$, is the expected relationship for a Poisson process. Notice that the measured variance typically exceeds the mean count. The example illustrated above (highlighted region of Fig. $1 A)$ is one of the rare exceptions. The variance is commonly described by a power law function of the mean count. The solid curve depicts the fit, Var $=0.4$ mean $^{1.3}$, but the fit is only marginally better than a simple proportional rule: $\mathrm{Var} \approx 1.3$ mean. Both the timing and count analyses suggest that the structured spike discharge apparent in the raster could be explained as a random point process with varying rate. The process is not exactly Poisson (e.g., the variance is too large), a point to which we shall return in detail. However, the key point is that the structure evident in the raster of Figure 1 is only a manifestation of a time-varying spike rate. The visual stimulus causes the neuron to modulate its spike rate consistently from trial to trial, whereas the timing of individual spikes-their intervals and patterns-is effectively random, hence best regarded as noise.

The neuron in Figure 1 illustrates the four properties of statistical homogeneity listed in Table 1: dynamic range, irregularity of the ISI, spike count variance, and the time course of spike rate modulation. As suggested above, it seems likely that these properties are characteristic of the neuron's afferent inputs and its output targets alike. Its dynamic range is typical of MT neurons, as well as of V1 neurons that project to MT (Movshon and Newsome, 1996) and neurons in MST (Celebrini and Newsome, 1994), a major target of MT projections. Indeed, neurons throughout the neocortex appear to be capable of discharging over a dynamic range of $\sim 0-200$ impulses/sec. Second, the ISIs from this neuron are characteristic of other neurons in its column and elsewhere in the visual cortex (Softky and Koch, 1993). Where it has been examined, the distribution of ISIs has a long "exponential" tail that is suggestive of a Poisson process. Third, the variance of the spike count of this neuron exceeds the mean by a margin that is typical of neurons throughout the visual cortex. Finally, the rapid modulation of the discharge rate occurs at a time scale that is on the order of an ISI of any one of its inputs. Our goal is to understand the basis of these statistical properties in single neurons and their conservation in networks of interconnected neurons.

\section{MATERIALS AND METHODS}

Physiology. Electrophysiological data (as in Fig. 1) were obtained by standard extracellular recording of single neurons in the alert rhesus monkey (Macaca mulatta). A full description of methods can be found in Britten et al. (1992). Experiments were in compliance with the National Institutes of Health guidelines for care and treatment of laboratory animals. The unit in Figure 1 was recorded from the middle temporal visual area (MT or V5). These trials were extracted from an experiment in which the monkey judged the net direction of motion of a dynamic random dot kinematogram, which was displayed for $2 \mathrm{sec}$ in the receptive field while the monkey fixated a small spot. In the particular trials shown in Figure 1, dots were plotted at high speed at random locations on the screen, resulting in a stochastic motion display with no net motion in any direction. Importantly, however, the exact pattern of random dots was repeated for each of the trials shown.

Model neuron. We performed computer simulations of neural discharge using a simple counting model of synaptic integration. Both excitatory and inhibitory inputs to the neuron are modeled as simple time series. With a few key exceptions, they are constructed as a sequence of spike arrival times with intervals that are drawn from an exponential distribution. The model neuron counts these inputs; when the count exceeds some threshold barrier, it emits an output spike and resets the count to zero. Each excitatory synaptic input increments the count by one unit step. The count decays to zero with a time constant, $\tau$, representing the membrane time constant or integration time constant. Each inhibitory input decrements the count by one unit. If the count reaches a negative barrier, however, it can go no further. Thus inhibition subtracts from any accumulated count, but it does not hyperpolarize the neuron beyond this barrier. Except where noted, we placed this reflecting barrier at the resting potential (zero) or one step below it.

Figure 2, $B, D$, and $F$, represents the count by the height of a particle. Excitation drives the particle toward an absorbing barrier at spike thresh- 

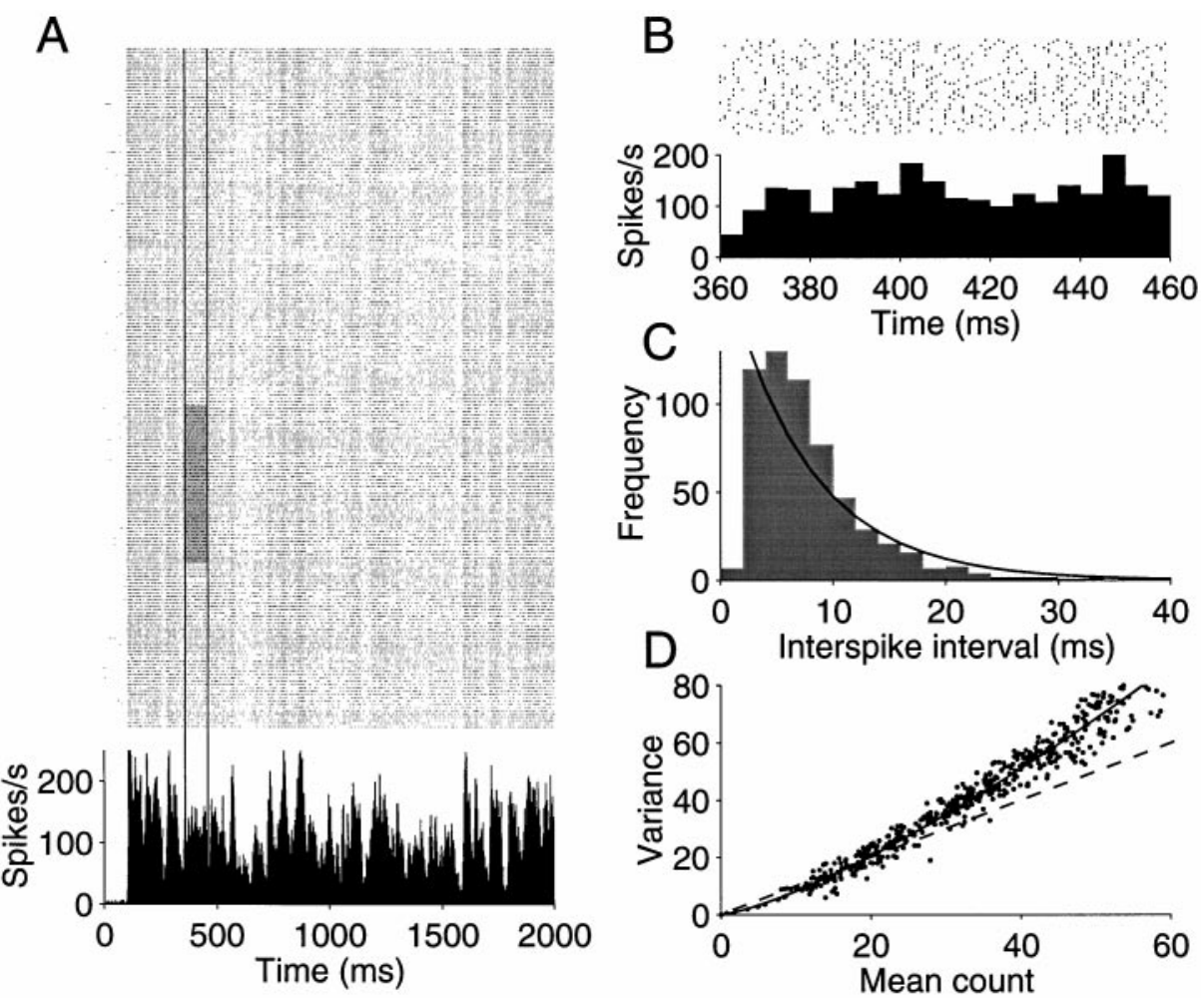

Figure 1. Response variability of a neuron recorded from area MT of an alert monkey. $A$, Raster and peristimulus time histogram (PSTH) depicting response for 210 presentations of an identical random dot motion stimulus. The motion stimulus was shown for $2 \mathrm{sec}$. Raster points represent the occurrence of action potentials. The PSTH plots the spike rate, averaged in 2 msec bins, as a function of time from the onset of the visual stimulus. The response modulates between 15 and 220 impulses/sec. Vertical lines delineate a period in which spike rate was fairly constant. The gray region shows 50 trials from this epoch, which were used to construct $B$ and $C$. $B$, Magnified view of the shaded region of the raster in $A$. The spike rate, computed in $5 \mathrm{msec}$ bins, is fairly constant. Notice that the magnified raster reveals substantial variability in the timing of individual spikes. $C$, Frequency histogram depicting the spike intervals in $B$. The solid line is the best fitting exponential probability density function. $D$, Variance of the spike count is plotted against the mean number of spikes obtained from randomly chosen rectangular regions of the raster in $A$. Each point represents the mean and variance of the spikes counted from 50 to 200 adjacent trials in an epoch from 100 to $500 \mathrm{msec}$ long. The shaded region of $A$ would be one such example. The best fitting power law is shown by the solid curve. The dashed line is the expected relationship for a Poisson point process. old, whereas inhibition drives the particle toward a reflecting barrier (represented by the thick solid line) just below zero. The particle represents the membrane voltage or the integrated current arriving at the axon hillock. The height of the absorbing barrier is inversely related to the size of an excitatory synaptic potential. It is the number of synchronous excitatory inputs necessary to depolarize the neuron from rest to spike threshold.

The model makes a number of simplifying assumptions, which are known to be incorrect. There are no active or passive electrical components in the model. We have ignored electrochemical gradients or any other factor that would influence the impact of a synaptic input on membrane polarization -with one exception. The barrier to hyperpolarization at zero is a crude implementation of the reversal potential for the ionic species that mediate inhibition. We have intentionally disregarded any variation in synaptic efficacy. All excitatory synaptic events count the same amount, and the same can be said of inhibitory inputs. Thus we are considering only those synaptic events that influence the postsynaptic neuron (no failures). We have ignored any variation in synaptic amplitude that would affect spikes arriving from the same input-because of adaptation, facilitation, potentiation, or depressionand we have ignored any differences in synaptic strength that would distinguish inputs. In this sense we have ignored the geometry of the neuron. We will justify this simplification in Discussion but state here that our strategy is conservative with respect to our aims and the conclusions we draw. Finally, we did not impose a refractory period or any variation that would occur on reset after a spike (e.g., afterhyperpolarization). The model rarely produces a spike within $1 \mathrm{msec}$ of the one preceding, so we opted for simplicity. Appendix 1 describes a more realistic model with several of the biophysical properties omitted here.

We have used this model to study the statistics of the output spike discharge. It is important to note that there is no noise intrinsic to the neuron itself. Consistent with experimental data (Calvin and Stevens, 1968; Mainen and Sejnowski, 1995; Nowak et al., 1997), all variability is assumed to reflect the integration of synaptic inputs. Because there are no stochastic components in the modeled postsynaptic neuron, the variability of the spike output reflects the statistical properties of the input spike patterns and the simple integration process described above.

A key advantage to the model is its computational simplicity. It enables large-scale simulations of synaptic integration under the assumption of dense connectivity. Thus a unique feature of the present exercise is to study the numerical properties of synaptic integration in a high-input regime, in which one to several hundred excitatory inputs arrive at the dendrite for every action potential the neuron produces.

\section{RESULTS}

\section{1: Problem posed by high-input regime}

Figure 2 illustrates three possible strategies for synaptic integration in the high-input regime. Figure $2 A$ depicts the spike discharge from 300 excitatory input neurons over a $100 \mathrm{msec}$ epoch. Each input is modeled as a random (Poisson) spike train with an average discharge rate of 50 impulses/sec (five spikes in the 100 msec epoch shown). The problem we wish to consider is how the postsynaptic neuron can integrate this input and yet achieve a reasonable spike rate. To be concrete, we seek conditions that allow the postsynaptic neuron to discharge at 50 impulses/sec. There is nothing special about the number 50, but we would like to conceive of a mechanism that produces a graded response to input over a range of $0-200$ spikes/sec. One way to impose this constraint is to identify conditions that would allow the neuron to respond at the average rate of any one of its inputs (that is, output spike rate should approximate the number of spikes per active input neuron per time).

A counting mechanism can achieve this goal through three types of parameter manipulations: a high absorption barrier (spike threshold), a short integration time (membrane time constant), or a balancing force on the count (inhibition). Figure 2 shows how each of these manipulations can lead to an output spike rate that is approximately the same as the average input. The simplest way to get five spikes out of the postsynaptic neuron is to impose a high spike threshold. Figure $2 B$ depicts the output from a simple integrate-and-fire mechanism when the threshold is set to 150 steps. Each synaptic input increments the count toward the absorption barrier, but the count decays with an integration time constant of $20 \mathrm{msec}$. The counts might be interpreted as voltage steps of $50-100 \mu \mathrm{V}$, pushing the membrane voltage from 
Figure 2. Three counting models for synaptic integration in the high-input regime. The diagrams $(B, D, F)$ depict three strategies that would permit a neuron to count many input spikes and yet produce a reasonable spike output. For each of the strategies, model parameters were adjusted to produce an output spike count that is the same, on average, as any one input. The membrane state is represented by a particle that moves between a lower barrier and spike threshold (top bar). The height of the particle reflects the input count. Each EPSP drives the particle toward spike threshold, but the height decays to the ground state with time constant, $\tau$ (insets). When the particle reaches the top barrier, an action potential occurs, and the process begins again with the count reset to 0 . $A$, Excitatory input to the model neurons. The 300 input spike trains are depicted as rows of a raster. Each input is modeled as a Poisson point process with a mean rate of 50 spikes/sec. The simulated epoch is $100 \mathrm{msec}$. $C, E, G$, Model response. The particle height is interpreted as a membrane voltage that is plotted as a function of time. These outputs were obtained using input spikes in $A$ and the model illustrated in the middle column $(B, D, F) . B$, $C$, Integrate-and-fire model with negligible inhibition and $20 \mathrm{msec}$ time constant. To achieve an output of five spikes in the $100 \mathrm{msec}$ interval, the spike threshold was set to 150 steps above the resting/reset state. Notice the regular interspike intervals in C.D, E, Coincidence detector. The spike threshold is only 16 steps above rest/reset, but the time constant must be $1 \mathrm{msec}$ to achieve five spikes out. The coincidence detector fires if and only if there is sufficient synchronous excitation. $F, G, \mathrm{Bal}-$ anced excitation-inhibition. A second set of inputs, like the ones shown in $A$, provide inhibitory input. Each inhibitory event moves the particle toward the lower barrier. The spike threshold is 15 steps above rest/reset, and the time constant is $20 \mathrm{msec}$. The particle follows a random walk, constrained by the lower barrier and the absorption state at spike threshold. This model is most consistent with known properties of cortical neurons. A more realistic implementation is described in Appendix 1.

its resting potential $(-70 \mathrm{mV})$ to spike threshold $(-55 \mathrm{mV})$. This textbook integrate-and-fire neuron (Stein, 1965; Knight, 1972) responds at approximately the same rate as any one of its 300 excitatory inputs. There are problems, however, that render this solution untenable. The mechanism grossly underestimates the impact of individual excitatory synaptic inputs (Mason et al., 1991; Otmakhov et al., 1993; Thomson et al., 1993a, b; Thomson and West, 1993), and it produces a periodic output spike train. The regularity of the spike output in Figure $2 C$ contrasts markedly with the random ISIs that constitute the inputs in Figure $2 A$. As suggested by Softky and Koch (1993), these observations are clear enough indication to jettison this mechanism.

If relatively few counts are required to reach the absorption barrier, then the synaptic integration process must incorporate an elastic force that pulls the count back toward the ground state. This can be accomplished by shortening the integration time constant or by incorporating a balancing inhibitory force that diminishes the count. Figure $2 D$ depicts a particle that steps toward the absorption barrier with each excitatory event. It takes only 16 steps to reach spike threshold, but the count decays
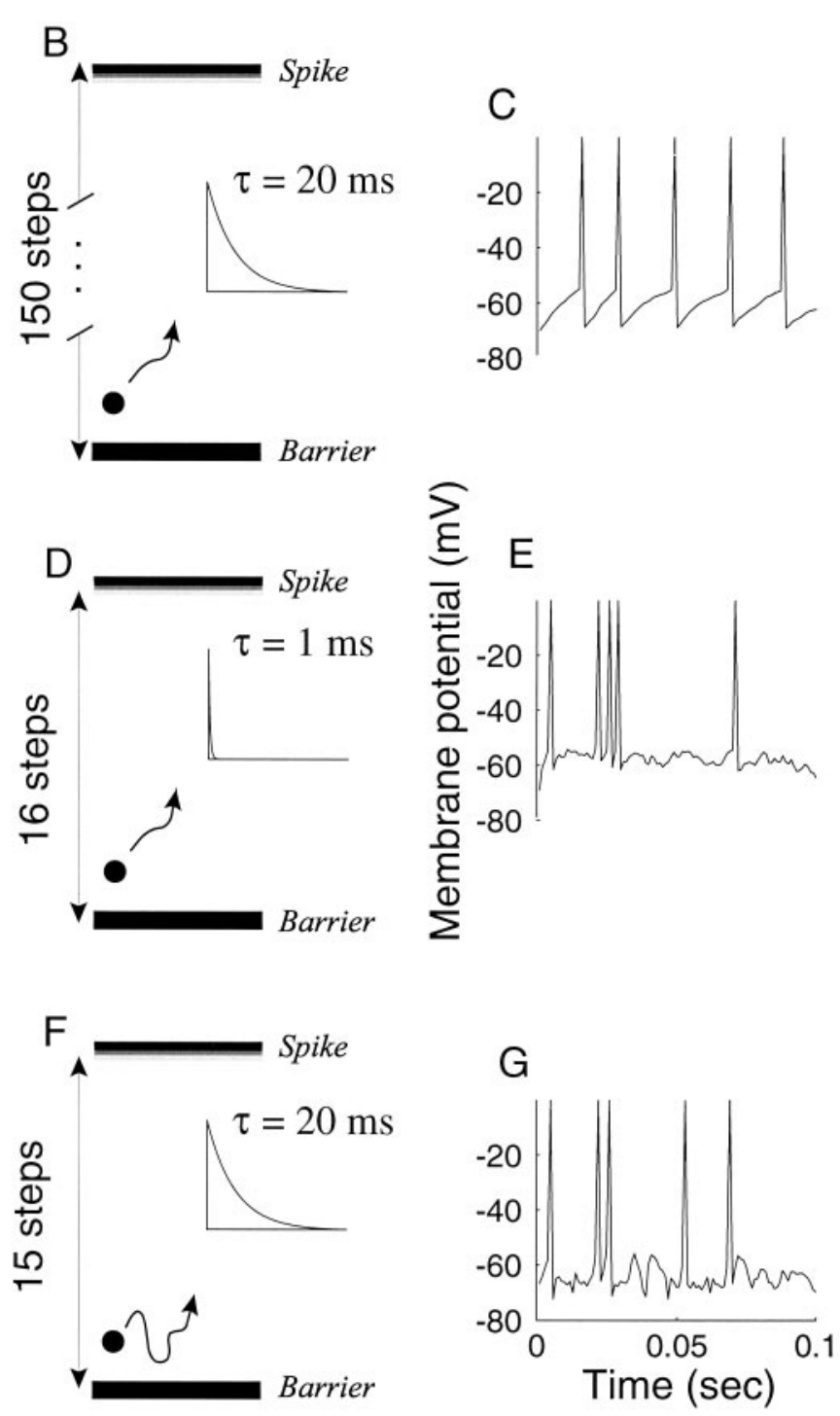

according to an exponential with a short time constant $(\tau=1$ $\mathrm{msec}$ ). There is no appreciable inhibitory input. The resulting output is shown in Figure $2 E$. The simulated spike train is quite irregular, reflecting occasional coincidences of spikes among the inputs. Because of the short time constant, the coincidences are sensed with precision well below the average interspike interval. Again, had we chosen a higher threshold, we could have achieved a proper spike output with a longer time constant, but only at the price of a regular ISI (even $3 \mathrm{msec}$ is too long). The mechanism illustrated in Figure 2, $D$ and $E$, detects coincidental synaptic input such that only the synchronous excitatory events are represented in the output spike train. Although the coincidence detector produces an irregular ISI, it requires an unrealistically short membrane time constant (Mason et al., 1991; Reyes and Fetz, 1993). This requirement can be relaxed somewhat when spike rates are low and the inputs are sparse (Abeles, 1982), but the mechanism is probably incompatible with the high-input regime considered in this paper. This is disappointing because this model would effectively time stamp presynaptic events that are sufficient to produce a spike, providing the foundation for propagation of a 
precise temporal code in the form of spike intervals (Abeles, 1991; Engel et al., 1992; Abeles et al., 1993; Softky, 1994; Konig et al., 1996; Meister, 1996).

The third strategy is to balance the excitation with inhibitory input. This is illustrated in the bottom panels of Figure 2. For each of the 300 excitatory inputs shown in Figure $2 A$, there is an equivalent amount of inhibitory drive (data not shown). Each excitatory synaptic input drives the particle toward the absorption barrier, as in Figure 2, $B$ and $C$; each inhibitory input moves the particle toward the ground state. The accumulated count decays with a time constant of $20 \mathrm{msec}$. The particle follows a random walk to the absorption barrier situated 15 steps away. The lower barrier just below the reset value crudely implements a synaptic reversal potential for the inhibitory current. The membrane potential is not permitted to fall below this value. In other words, inhibitory synaptic input is only effective when the membrane is depolarized from rest.

This model is an integrate-and-fire neuron with balanced excitation and inhibition. It implies that the neuron varies its discharge rate as a consequence of harmonious changes in its excitatory and inhibitory drive. Conditions that lead to greater excitation also lead to greater inhibition. This idea is reasonable because most of the inhibitory input to neurons arises from smooth stellate cells within the same cortical column (Somogyi et al., 1983a; DeFelipe and Jones, 1985; Somogyi, 1989; Beaulieu et al., 1992). Thus excitatory and inhibitory inputs are activated by the same stimuli; e.g., they share the same preference for orientation (Ferster, 1986), or they are affected similarly by somatosensory stimulation (Carvell and Simons, 1988; McCasland and Hibbard, 1997). Contrast this idea with the standard concept of a push-pull arrangement in which the neural response reflects the degree of imbalance between excitation and inhibition. In the high-input regime, more inhibition is needed to balance the excitatory drive. The balance confers a proper firing rate without diminishing the impact of single EPSPs or the membrane time constant (but see Appendix 1). The cost, however, is an irregular ISI. Gerstein and Mandelbrot (1964) first proposed that such a process would give rise to an irregular ISI, and numerous investigators have implemented similar strategies, termed random walk or diffusion models (Ricciardi and Sacerdote, 1979; Lansky and Lanska, 1987; for review see Tuckwell, 1988). What is novel in our analysis is that the same idea allows the neuron to respond over the same dynamic range as any one of its many inputs. That is, it allows the neuron to operate in a high-input regime. This simple idea has important implications for the propagation of signal and noise through neural networks of the neocortex.

\section{2: Dynamic range}

The counting model with balanced excitation and inhibition achieves a proper dynamic range of response using reasonable parameters. Figure $3 A$ shows the response of a model neuron as a function of the average response of the inputs. We used 300 excitatory and 300 inhibitory inputs in these simulations. The output response is nearly identical to the response of any one input, on average. This neuron is performing a very simple calculation, averaging, but it is doing so in a high-input regime. Consider that there are $\sim 300$ excitatory synaptic inputs for every spike, yet each excitatory input delivers $1 / 15$ the depolarization necessary to reach spike threshold. By balancing the surfeit of excitation with a similar inhibitory drive, the neuron effectively compresses a large number of presynaptic events into a more manageable number of spikes. Sacrificed are details about the

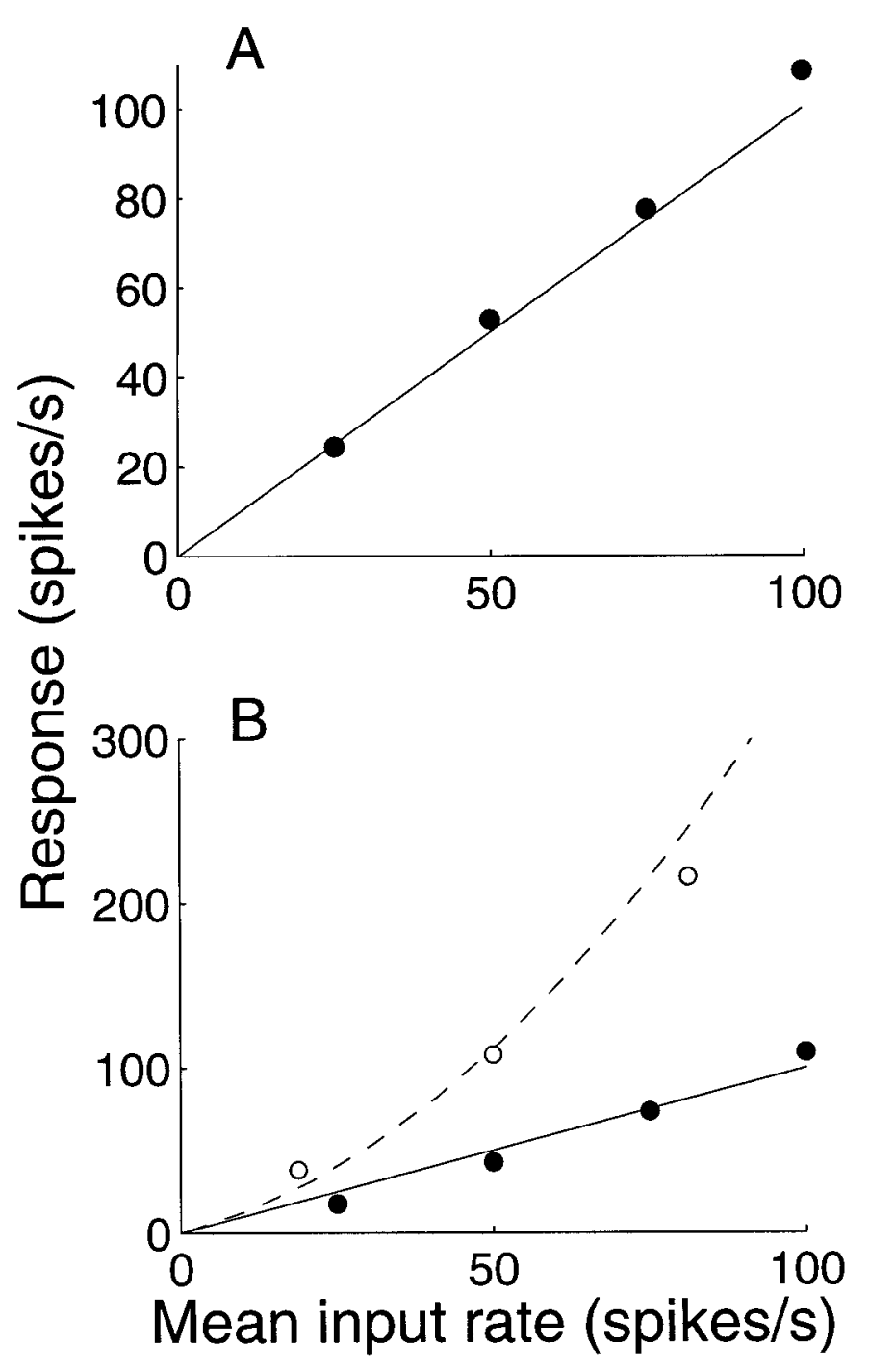

Figure 3. Conservation of response dynamic range. The spike rate of the model neuron is plotted as a function of the average input spike rate. $A$, Simulations with 300 excitatory inputs and 300 inhibitory inputs; parameters are the same as in Figure 2, $F$ and $G$ (barrier height, 15 steps; $\tau=$ $20 \mathrm{msec}$ ). The balanced excitation-inhibition model produces a response that is approximately the same as one of its many inputs. $B$, Simulations with 600 excitatory and inhibitory inputs. Open symbols and dashed curve show the response obtained using the same model parameters as in $A$. Solid symbols and curve show the response when the barrier height is increased to 25 steps. These simulations suggest that a small hyperpolarization could be applied to enforce a unity gain input-output relationship when the number of active inputs is large.

input spike times; they are only reflected in the tiny bumps and wiggles that describe the membrane voltage during the interspike interval. This capacity for compression permits the neuron to integrate inputs from its dendrites and thus to perform calculations on large numbers of inputs.

The mechanism should also allow the neuron to adapt to a broad range of activation in which more or fewer inputs are active. Figure $3 B$ shows the results of simulations using twice the number of excitatory and inhibitory inputs. The dashed curve depicts the model response using the identical parameters to those in Figure $3 A$. The output response is now a little larger than the average input, and the relationship is approximately quadratic. The departure from linearity is attributable to the mem- 
brane time constant. At higher input rates, the count frequently accumulates toward spike threshold before there is any time to decay. Although the range of response is reasonable, it is not a sustainable solution. If every neuron were to exhibit such amplification, the response would exceed the observed dynamic range in very few synapses. Imagine a chain of neurons, each squaring the response of its averaged input.

A small adjustment to the model repairs this. The solid curve in this graph was obtained after changing the height of the threshold barrier from 15 to 25 . The neuron can now accommodate a doubling of the number of inputs. With what amounts to a few millivolts of hyperpolarization, the neuron can achieve substantial control of its gain. Such a mechanism has been shown to underlie the phenomenon of contrast adaptation in visual cortical neurons (Carandini and Ferster, 1997). In addition, the curves in Figure $3 B$ raise the possibility that a neuron could compute the square of a quantity by a small adjustment in its resting membrane potential or conductance. This observation may be relevant to computational models that use squaring-type nonlinearities (Adelson and Bergen, 1985; Heeger, 1992a).

Our central point is that a simple counting model can accommodate large numbers of inputs with relatively modest adjustment of parameters. It is essential, however, that a balance of inhibition holds. Because excitatory synapses typically outnumber inhibitory inputs by about 6:1 for cortical neurons (Somogyi, 1989; Braitenberg and Schüz, 1991), it is possible that the control of excitation (e.g., presynaptic release probability or synaptic depression) may play a role in maintaining the balance (Markram and Tsodyks, 1996; Abbott et al., 1997).

\section{3: Irregularity of the interspike interval}

As indicated in the preceding section, a consequence of the balanced excitation-inhibition model is an irregular ISI. Figure $4 A$ shows a representative interval histogram for one simulation. The intervals were collated from $20 \mathrm{sec}$ of simulated response at a nominal rate of 50 spikes/sec. The solid curve is the best fitting exponential probability density function.

The variability of the ISI is commonly measured by its coefficient of variation $\left(C_{\mathrm{V}_{\mathrm{ISI}}}=\mathrm{SD} /\right.$ mean $)$. The value for the example in Figure $4 A$ is 0.9 , just less than the value expected of a random process (for an exponential distribution, $C_{\mathrm{V}_{\mathrm{ISI}}}=1$ ). The value is typical for these simulations, appearing impervious to spike rate or the number of inputs. Figure $4 B$ shows the distribution of $C_{\mathrm{V}_{\text {ISI }}}$ obtained for 128 simulations incorporating a variety of parameters including those used to produce Figure 3 (solid symbols). The simulations encompassed a broad range of spike rates, but all produced an irregular spike output. The $C_{\mathrm{V}_{\mathrm{ISI}}}$ of $0.8-0.9$ reflects a remarkable degree of variation in the ISI. Because the model is effectively integrating the response from a very large number of neurons, one might expect such a process to effectively "clean up" the irregularity of the inputs, as in Figure $2 C$ (Softky and Koch, 1993). The irregularity is a consequence of the balance between excitation and inhibition, suggesting an analogy between the ISI and the distribution of first passage times of random-walk (diffusion) processes.

In our simple counting model, the relationship between input and output spikes is entirely deterministic. All inputs affect the neuron with the same strength, and there is no chance for an input to fail. The only source of irregularity in the model is the time of the input spikes themselves. We simulated the input spike trains as random point processes, and the counting mechanism nearly preserved the exponential distribution of ISIs in its output.
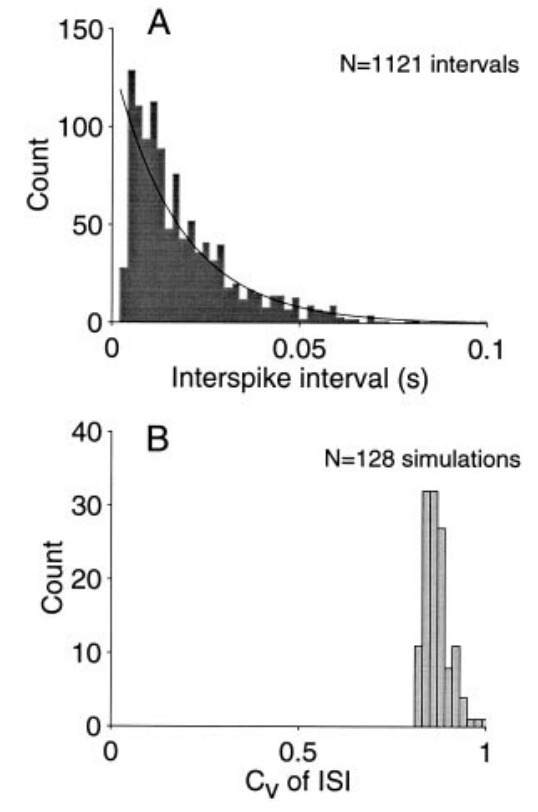

Figure 4. Variability of the interspike interval. A, Frequency histogram of ISIs from one simulation using 300 inputs at 50 spikes/sec. Notice the substantial variability. The SD divided by the mean interval is known as the coefficient of variation of the interspike interval $\left(C_{\mathrm{V}_{\mathrm{ISI}}}\right)$. The value for this simulation is 0.9 . The distribution is approximated by an exponential probability density (solid curve), which would predict $C_{\mathrm{V}_{\mathrm{ISI}}}=1$. B , Coefficient of variation of the interspike interval $\left(C_{\mathrm{V}_{\text {ISI }}}\right)$ from 128 simulations using 300 and 600 inputs and a variety of spike rates. Each simulation generated $20 \mathrm{sec}$ of spike discharge using parameters that led to a similar rate of discharge for input and output neurons (i.e., a common dynamic range). The average $C_{\mathrm{V}_{\mathrm{ISI}}}$ was 0.87 .

But it did not do so completely; the $C_{\mathrm{V}_{\mathrm{ISI}}}$ was slightly $<1$. This raises a possible concern. To what extent does the output spike irregularity depend on our choice of inputs? Suppose the input spike trains are more regular than Poisson spike trains; suppose they are only as irregular as the spike trains produced by the model. Would the counting mechanism reduce the irregularity further?

Figure 5 shows the results of a series of simulations in which we varied the statistics of the input spike trains. We used the same simulation parameters as in Figure $3 A$ but constructed the input spike trains by drawing ISIs from families of $\gamma$ distributions which lead to greater or less irregular intervals than the Poisson case (Mood et al., 1963). By varying the parameters of the distribution we maintained the same input rate while affecting the degree of irregularity of the spike intervals. Figure 5 plots the $C_{\mathrm{V}_{\mathrm{ISI}}}$ of our model neuron as a function of the $C_{\mathrm{V}_{\mathrm{ISI}}}$ for the inputs. The Poisson-like inputs would have a $C_{\mathrm{V}_{\mathrm{ISI}}}$ of 1 . Notice that for a wide range of input $C_{\mathrm{V}_{\mathrm{ISI}}}$, the output of the counting model attains a $C_{\mathrm{V}_{\text {ISI }}}$ that is quite restricted and relatively large. The fit intersects the main diagonal at $C_{\mathrm{V}_{\mathrm{ISI}}}=0.8$. This implies that the mechanism would effectively randomize more structured input and tend to regularize (slightly) a more irregular input. Most importantly, the result indicates that the output irregularity is not merely a reflection of input spike irregularity. The irregular ISI is a consequence of the balanced excitation-inhibition model.

These ideas are consistent with Calvin and Steven's (1968) seminal observations in motoneurons that the noise affecting spike timing is attributable to synaptic input rather than stochastic properties of the neuron itself (e.g., variable spike threshold) 


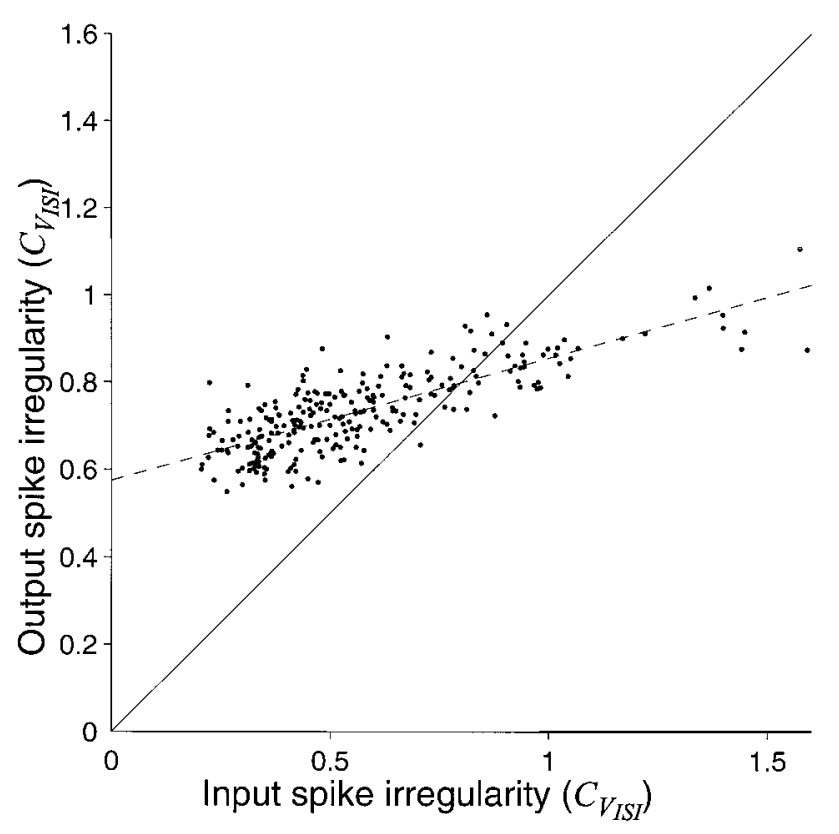

Figure 5. Irregularity of the spike discharge is not merely a reflection of input spike irregularity. The graph compares the irregularity of the ISI produced by the balanced excitation-inhibition model with the irregularity of the intervals constituting the 300 excitatory and inhibitory input spike trains. The input spike trains were constructed by drawing intervals randomly from a gamma distribution. By varying the parameters of the gamma distribution, the input $C_{\mathrm{V}_{\mathrm{ISI}}}$ was adjusted from relatively regular to highly irregular (abscissa). Each point represents the results of one simulation, using different parameters for the input interval distribution. Notice that the degree of input irregularity has only a weak effect on the distribution of output interspike intervals. Points above the main diagonal represent simulations in which the counting model produced a more irregular discharge than the input spike trains. Points below the main diagonal represent simulations in which the output is less irregular than the input spike trains. The dashed line is the least squares fit to the data. This line intersects the main diagonal at $C_{\mathrm{V}_{\mathrm{ISI}}}=0.8$. The best fitting line does not extrapolate to the origin, because the inputs are not necessarily synchronous.

(Calvin and Stevens, 1968; Mainen and Sejnowski, 1995; Nowak et al., 1997). Nonetheless, if the random walk to a barrier offers an adequate explanation of ISI variability, then it is natural to view the irregular ISI as a signature of noise and to reject the notion that it carries a rich temporal code. The important insight is that the irregular ISI may be a consequence of synaptic integration and yet may reflect little if any information about the temporal structure of the synaptic inputs themselves (Shadlen and Newsome, 1994; van Vreeswijk and Sompolinsky, 1996).

\section{4: Variance of spike count}

It is important to realize that the coefficient of variation that we have calculated is an idealized quantity. It rests on the assumption that the input rates are constant and that the input spike trains are uncorrelated. Under these assumptions the number of input spikes arriving in any epoch would be quite precise. For example, at an average input rate of 100 spikes/sec, the number of spikes arising over the ensemble of 300 inputs varies by only $2 \%$ in any $100 \mathrm{msec}$ interval. Variability produced by the model is therefore telling us how much noise the neuron would add to a simple computation (e.g., the mean) when the solution ought to be the same in any epoch. This will turn out to be a useful concept (see section 3 below), but it is not anything that we can actually measure in a living brain. In reality, inputs are not independent,

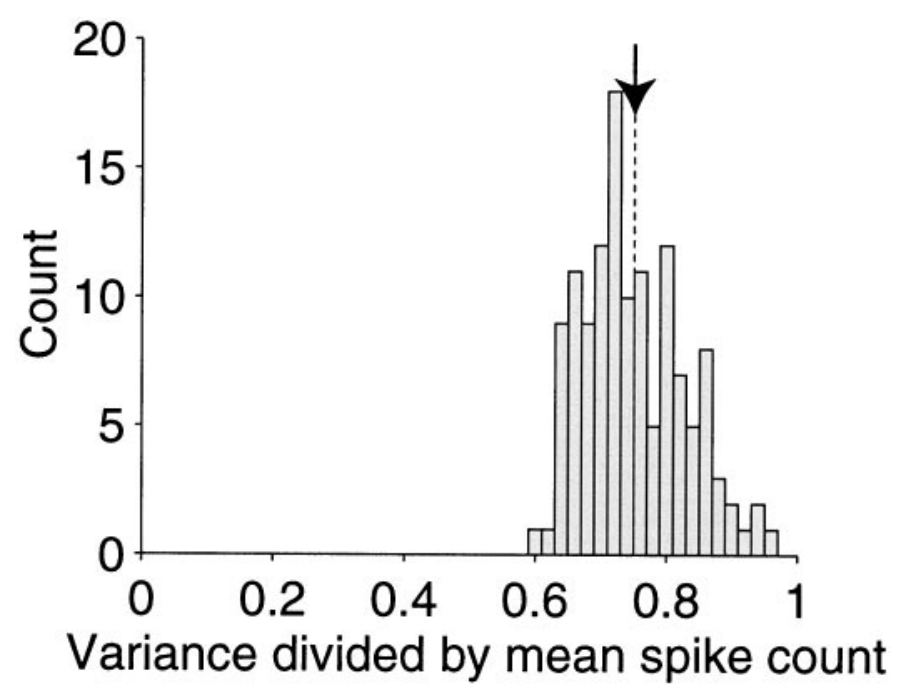

Figure 6. Frequency histogram of the spike count variance-to-mean ratios obtained from the same simulations as in Figure $4 B$. For each of the simulations, the spikes were counted in 200 epochs of $100 \mathrm{msec}$ duration. The variance in the number of spikes produced by the model in each of these epochs is proportional to the mean of the counts obtained for these epochs. Spike count variability is therefore conveniently summarized by the variance-to-mean ratio. The average ratio is 0.75 (arrow).

and the number of spikes among the population of inputs would be expected to be more variable. We will attach numbers to these caveats in subsequent sections. For now, it is interesting to calculate one more idealized quantity.

If, over repeated epochs, the number of input spikes were indeed identical (or nearly so), how would the spike count of the output neuron vary over repeated measures? Using the same simulations as in Figures 3 and 4, we divided each $20 \mathrm{sec}$ simulation into 200 epochs of 100 msec. We computed the mean and variance of the spikes counted in these epochs and calculated the ratio: variance/mean. Figure 6 shows the distribution of variance/ mean ratios for a variety of spike output rates and model parameters. The ratios are concentrated between 0.7 and 0.8 , just slightly less than the value expected for a random Poisson point process.

There are two salient points. First, notice that the histogram of variance/mean ratios appears similar to the histogram of $C_{\mathrm{V}_{\mathrm{ISI}}}$ from the same simulations (Fig. $4 B$ ). In fact, the ratios in Figure 6 are approximated by squaring the values for $C_{\mathrm{V}_{\mathrm{ISI}}}$ in Figure $4 B$. This is a well known property of interval and count statistics for a class of stochastic processes known as renewals (Smith, 1959). We will elaborate this point in section 3. Second, the variance/ mean ratios fall short of the value measured in visual cortex (i.e., 1-1.5). Clearly the variability observed in vivo reflects sources of noise beyond the mechanisms we have considered. In contrast to our simulations, a real neuron does not receive an identical number of input spikes in each epoch; the input is itself variable. A key part of this variability arises from correlation among the inputs. In the next section we turn attention to properties of cortical neurons that lead to correlated discharge. We will return to the issue of spike count variance in section 3 .

\section{2: Redundancy, correlation, and signal fidelity}

The preceding considerations lead us to depict the neuronal spike train as a nearly random realization of an underlying rate term reflecting the average input spike rate (i.e., the number of input 
spikes per input neuron per time), or some calculation thereon. Whether we accept this argument on principle, there is little doubt that many cortical neurons indeed transmit information via changes in their rate of discharge. Yet, the irregular ISI precludes single neurons from transmitting a reliable estimate of this very quantity. Because the spike count from any one neuron is highly variable, several ISIs would be required to estimate the mean firing rate accurately (Konig et al., 1996). The irregular ISI therefore poses an important constraint on the design of cortical architecture: to transmit rate information rapidly-say, within a single ISI-several copies of the signal must be transmitted. In other words, the cortical design must incorporate redundancy. In this section we will quantify the notion of redundancy and explore its implications for the propagation of signal and noise in the cortex.

\section{1: Redundancy necessitates shared connections}

By redundancy we refer to a group of neurons, each of which encodes essentially the same signal. Ideally, each neuron would transmit an independent estimate of the signal through its rate of discharge. If the variability of the spike trains were truly uncorrelated (independent), then an ensemble of neurons could convey, in its average, a high-fidelity signal in a very short amount of time (e.g., a fraction of an ISI; see below). Although this is a desirable objective, the assumption of independence is unlikely to hold in real neural systems. Redundancy implies that cortical neurons must share connections and thus a certain amount of common variability.

The need for shared connections is illustrated in Figure $7 A$. The flow of information in this figure is from the bottom layer of neurons to the top. The neurons at the top of the diagram represent some quantity, $\gamma$. Many neurons are required to represent $\gamma$ accurately, because the discharge from any one neuron is so variable. To compute its estimate of $\gamma$, each neuron in the upper layer requires an estimate of some other quantity, $\beta$, supplied by the neurons in middle tier of the diagram. To compute $\gamma$ rapidly, however, each neuron at the top of the diagram must receive many samples of $\beta$. Note, however, that to compute $\beta$, each of the neurons in the middle of the diagram needs an estimate of some other quantity, $\alpha$. What was said of the neurons at the top applies to those in the middle panel as well. Thus each $\beta$ neuron must receive inputs from many $\alpha$ neurons. The chain of processing resembles a pyramid and is clearly untenable as a model for how neurons deep in the CNS come to encode any particular quantity. We cannot insist on geometrically large numbers of independent, lower-order neurons to sustain the responses of a higher-order neuron positioned a few synaptic links away. From this perspective, shared connectivity is necessary to achieve redundancy, and hence rapid processing, in a system of noisy neurons.

In Figure $7 B$, the same three tiers are illustrated, but the neurons encoding $\gamma$ receive some input in common. Each neuron projects to many neurons at the next stage. Viewed from the top, some fraction of the inputs to any pair of neurons is shared. In principle, a shared input scheme, such as the one in Figure $7 B$, would permit the cortex to represent quantities in a redundant fashion without requiring astronomical numbers of neurons. There is, however, a cost. If the neurons at the top of the diagram receive too much input in common, the trial-to-trial variation in their responses will be similar; hence the ensemble response will be little more reliable than the response of any single neuron.

We therefore wish to explore the influence of shared inputs on the responses of two cortical neurons such as the ones shaded at the top of Figure $7 B$. How much correlated variability results from differing amounts of shared input? How does correlated variability among the input neurons themselves (as in the middle tier of Fig. $7 B$ ) influence the estimate of $\gamma$ at the top tier? To what extent does shared connectivity lead to synchronous action potentials among neurons at a given level? How does synchrony among inputs influence the outputs of neurons in higher tiers? We can use the counting model developed in the previous section to explore these questions. Our goal is to clarify the relationship among common input, synchronous spikes, and noise covariance. A useful starting point is to consider the effect of shared inputs on the correlation in spike discharge from pairs of neurons.

\section{2: Shared connections lead to response correlation}

We simulated the responses from a pair of neurons like the ones shaded at the top of Figure $7 B$. Each neuron received 100-600 excitatory inputs and the same number of inhibitory inputs. A fraction of these inputs were identical for the pair of neurons. We examined the consequences of varying the fraction of shared inputs on the output spike trains. Except for this manipulation, the model is the same one used to produce the results in Figures 3 and 4. Thus each neuron responded approximately at the average rate of its inputs. We now have a pair of spike trains to analyze, and once again we are interested in interval and count statistics. For a pair of neurons, interval statistics are commonly summarized by the cross-correlation spike histogram (or crosscorrelogram); count statistics have their analogy in measures of response covariance. We will proceed accordingly.

Figure 8 depicts a series of cross-correlograms (CCGs) obtained for a variety of levels of common input. We obtained these functions from $20 \mathrm{sec}$ of simulated spike trains using the same parameters as in Figure $3 A$. The normalized cross-correlogram depicts the relative increase in the probability of obtaining a spike from one neuron, given a spike from the second neuron, at a time lag represented along the abscissa (Melssen and Epping, 1987; Das and Gilbert, 1995b). The probabilities are normalized to the expectation given the base firing rate for each neuron. Two observations are notable. First, the narrow central peak in the CCG reflects the amount of shared input to a neuron, as previously suggested (Moore et al., 1970; Fetz et al., 1991; Nowak et al., 1995). Second, no structure is visible in the correlograms until a rather substantial fraction of the inputs are shared. This is despite several simplifications in the model that should boost the effectiveness of correlation. For example, introducing variation in synaptic amplitude attenuates the correlation. Thus it is likely that the modest peak in the correlation obtained with $40 \%$ shared excitatory and inhibitory inputs represents an exaggeration of the true state of affairs.

Rather than viewing the entire CCG for each combination of shared excitation and inhibition, we have integrated the area above the baseline and used it to derive a simpler scalar value:

$$
r_{\mathrm{c}}=\frac{A_{12}}{\sqrt{A_{11} A_{22}}},
$$

where $A_{11}$ and $A_{22}$ represent the area under the normalized autocorrelograms for neurons 1 and 2, respectively, and $A_{12}$ is the area under the normalized cross-correlogram (the autocorrelogram is the cross-correlogram of one neural spike train with itself). The value of $r_{\mathrm{c}}$ reflects the strength of the correlation between the two neurons on a scale from -1 to 1 . This value is 

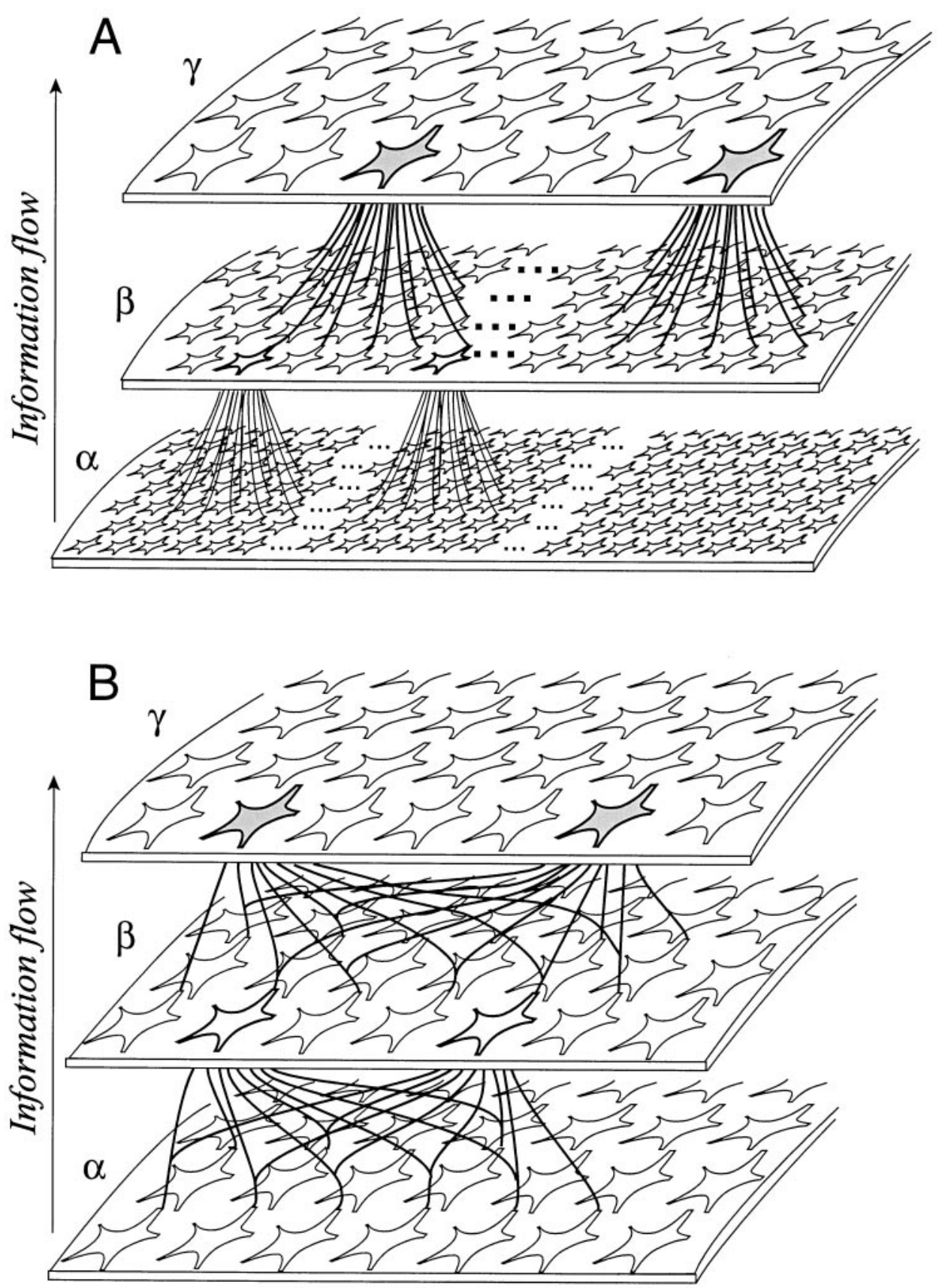

Figure 7. Redundancy necessitates shared connections. Three ensembles of neurons represent the quantities $\alpha, \beta$, and $\gamma$. Each neuron that represents $\gamma$ receives input from many neurons that represent $\beta$, and each neuron that represents $\beta$ receives input from many neurons that represent $\alpha$. $A$, There are no shared connections; each neuron receives a distinct set of inputs from its neighbor. The shaded neurons receive no common input, and the same can be said of any pair of neurons in the ensemble that represents $\beta$. The scheme would require an inordinately large number of neurons. $B$, Neurons share a fraction of their inputs. The shaded neurons receive some of the same inputs from the ensemble that represents $\beta$. Likewise, any pair of neurons in the $\beta$ ensemble receive some common input from the neurons that represent $\alpha$. This architecture allows for redundancy without necessitating immense numbers of neurons. Neither the number of neurons nor the number of connections are drawn accurately. Simulations suggest that the pair of shaded neurons might receive as much as $40 \%$ common input, and each needs about 100 inputs to compute with the quantity $\beta$.

equivalent to the correlation coefficient that would be computed from pairs of spike counts obtained from the two neurons across many stimulus repetitions (W. Bair, personal communication). ${ }^{a}$ It provides a much simpler measure of correlation than the entire CCG function.

${ }^{a}$ We were advised of this relationship by H. Sompolinsky and W. Bair. The area of the correlogram is:
Using $r_{\mathrm{c}}$ we can summarize the effect of shared excitation and

$$
A_{\mathrm{jk}}=\sum_{\tau=-50}^{50} \Theta(\tau)\left[C_{\mathrm{jk}}(\tau)-1\right],
$$

where $\Theta(\tau)=T-|\tau|$ is a triangular weighting function with a peak that lies at the center of the trial epoch of duration, $T$ msec, and 


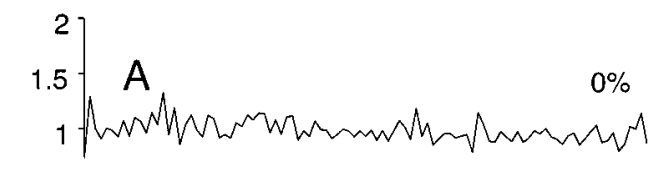

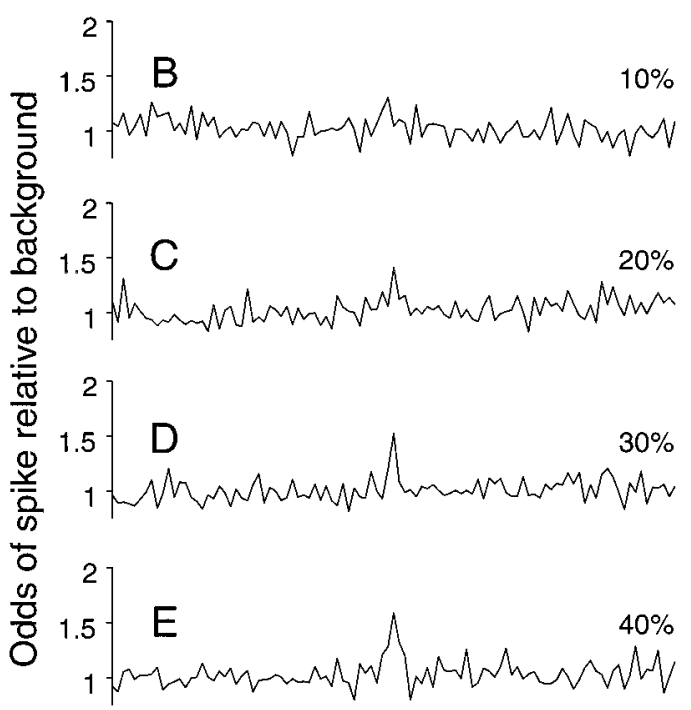

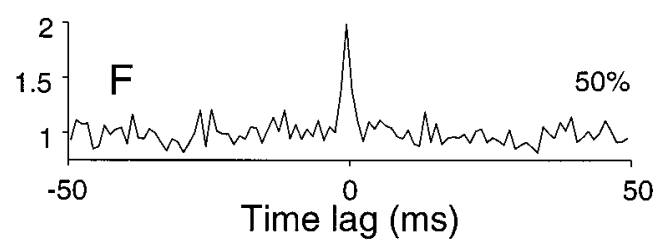

Figure 8. Cross-correlation response histograms from a pair of simulated neurons. The correlograms represent the relative change in response from one neuron when a spike has occurred in the other neuron at a time lag indicated along the abscissa. The spike train for each neuron was simulated using the random walk counting model with 300 excitatory and 300 inhibitory inputs. Plots $A-F$ differ in the amount of common input that is shared by the simulated pair. A small central peak in the correlogram is apparent when the pair of neurons share $20-50 \%$ of their inputs.

inhibition in a single graph. Figure 9 is a plot of $r_{\mathrm{c}}$ as a function of the fraction of shared excitatory and shared inhibitory inputs. The points represent correlation coefficients from simulations using 100, 300, and 600 excitatory and inhibitory inputs and a variety of spike rates. The threshold barrier was adjusted to confer a reasonable dynamic range of response (input spike rate divided by output spike rate was $0.75-1.5$ ). Over the range of simulated values, the correlation coefficient is approximated by the plane:

$$
r_{\mathrm{c}}=0.36 \phi_{\mathrm{E}}+0.22 \phi_{\mathrm{I}},
$$

where $\phi_{\mathrm{E}}$ and $\phi_{\mathrm{I}}$ are the fraction of shared excitatory and inhibitory inputs, respectively. The graph shows that both the fractions

$$
C_{\mathrm{jk}}(\tau)=\frac{1}{\lambda_{\mathrm{j}} \lambda_{\mathrm{k}} \Theta(\tau)}\left\langle\sum_{i=0}^{T-1} x_{\mathrm{j}}(i) x_{\mathrm{k}}(i+\tau)\right\rangle_{\text {(trials) }}
$$

is the normalized cross- or autocorrelation function computed from bins of binary values, $x_{\mathrm{j} \mid \mathrm{k}}(i)$, denoting the presence or absence of a spike in the $i^{\text {th }}$ millisecond from neuron $j$ or $k$. Mathematical details and a proof of Equation 1 will appear in a paper by E. Zohary, W. Bair, and W. T. Newsome (unpublished data).

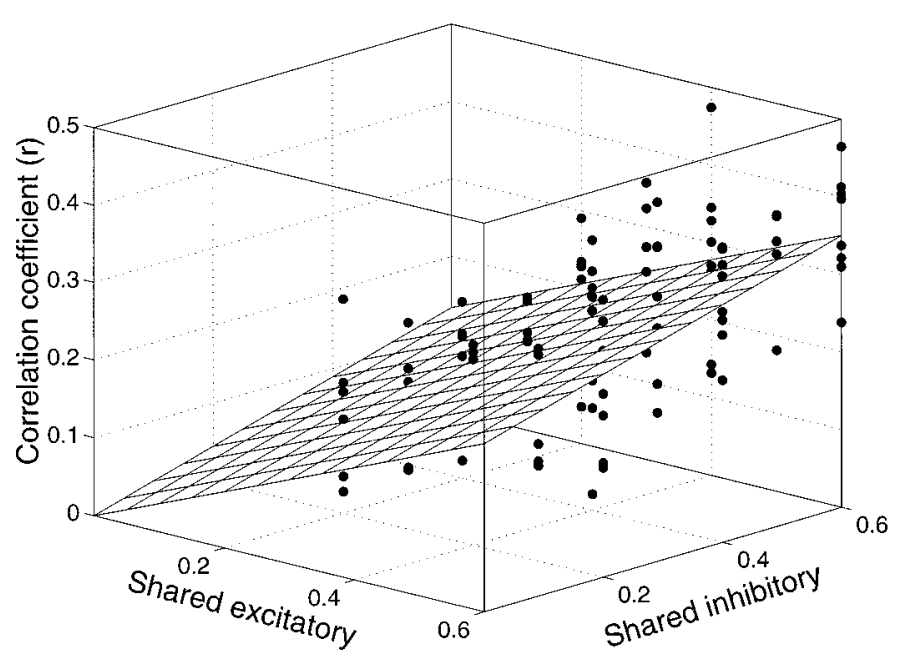

Figure 9. Effect of common input on response covariance. The correlation coefficient is plotted as a function of the fraction of shared excitatory and shared inhibitory input to a pair of model neurons. Each point was obtained from $20 \mathrm{sec}$ of simulated spike discharge using a variety of model parameters (input spike rate, number of inputs, and barrier height). In each simulation, the output spike rate was approximately the same as the average of any one input (within a factor of \pm 0.25 ). The best fitting plane through the origin is shown. A substantial degree of shared input is required to achieve even modest correlation.

of shared excitatory and shared inhibitory connections affect the correlation coefficient. Shared excitation has a greater impact, because it can lead directly to a spike from both neurons.

Over the range of counting model parameters tested, we find this planar approximation to be fairly robust (the fraction of variance of $r_{\mathrm{c}}$ accounted for by Eq. 2 is $42 \%$ ). We can improve the fit with a more complicated model (e.g., spike rate has a modest effect), but such detail is unimportant for the exercise at hand. Of course, Equation 2 must fail as the fraction of shared input approaches 1; the two neurons will follow identical random walks to spike threshold, and the correlation coefficient must therefore approach 1.

The most striking observation from Figure 9 is that only modest correlation is obtained when nearly half of the inputs are identical. The counting model is impressively resilient to common input, especially from inhibitory neurons. Electrophysiological recordings in visual cortex indicate that adjacent neurons covary weakly from trial to trial on repeated presentations of the same visual stimulus, with measured correlation coefficients typically ranging from 0.1 to 0.2 (van Kan et al., 1985; Gawne and Richmond, 1993; Zohary et al., 1994). The counting model suggests that such modest correlation might entail rather substantial common input, $\sim 30 \%$ shared connections, by Equation 2. This is larger than the amount of common input that might be expected from anatomical considerations. The probability that a pair of nearby neurons receive an excitatory synapse from the same axon is believed to be $\sim 0.09$ (Braitenberg and Schüz, 1991; Hellwig et al., 1994). Comparable estimates are not known for the axons from inhibitory neurons, although the probability is likely to be considerably larger (Thomson et al., 1996), because there are fewer inhibitory neurons to begin with. Still, it is unlikely that pairs of neurons share $50 \%$ of their inhibitory input; yet this is the value for $\phi_{\mathrm{I}}$ needed to attain a correlation of 0.15 (when $\phi_{\mathrm{E}}=$ 0.09 , Eq. 2). We suspect that this discrepancy arises in part because the covariation measured electrophysiologically exists 
not only because of common input to a pair of neurons at the anatomical level, but also because the signals actually transmitted by the input neurons are contaminated by common noise arising at earlier levels of the system. As an extreme example, small eye movements could introduce shared variability among all neurons performing similar visual computations (Gur et al., 1997).

\section{3: Response correlation limits fidelity}

Why should we care about such modest correlation? The reason is that even weak correlation severely limits the ability of the neural population to represent a particular quantity reliably (Johnson, 1980; Britten et al., 1992; Seung and Sompolinsy, 1993; Abbott, 1994; Zohary et al., 1994; Shadlen et al., 1996). Importantly for our present purposes, developing an intuition for this principle will help us understand a major component of the variability in the discharge of a cortical neuron.

Consider one of the shaded neurons shown in the top tier of Figure $7 B$. Its rate of discharge is supposed to represent the result of some computation involving the quantity $\beta$. For present purposes we need not worry about exactly what the neuron is computing with this value. What is important is that in any epoch, all that the shaded neuron knows about $\beta$ is the number of spikes it receives from neurons in the middle tier of Figure $7 B$. Clearly, the variability of the shaded neuron's spike output depends to some extent on the variability of the number of input spikes, no matter what the neuron is calculating. If the shaded neuron receives input from hundreds of neurons, each contributing an independent estimate of $\beta$, then the number of input spikes per neuron per unit time would vary minimally. For example, suppose that some visual stimulus contains a feature represented by the quantity $\beta=$ 40 spikes/sec. Each of the neurons representing this quantity would be expected to produce four spikes in a $100 \mathrm{msec}$ epoch, but because the spike train of any neuron is highly variable, each produces from zero to eight spikes. This range reflects the $95 \%$ confidence interval for a Poisson process with an expected count of four. We might say that the number of spikes from any one neuron is associated with an uncertainty of $50 \%$ (because the SD is two spikes; we use the term uncertainty here, rather than coefficient of variation, to avoid conf usion with $C_{\mathrm{V}_{\mathrm{ISI}}}$ ). In contrast, the average number of spikes from 100 independent neurons should almost always fall between 3.6 and 4.4 spikes per input (i.e., $\pm 2 \mathrm{SE}$ of the mean). The shaded neuron would receive a fairly reliable estimate of $\beta$, which it would incorporate into its calculation of $\gamma$. In this example, the uncertainty associated with the average input spike count is $5 \%$, that is, a 10 -fold reduction because of averaging from 100 neurons. With more neurons, the uncertainty can be further reduced, as illustrated by the gray line $(r=0)$ in Figure 10.

Unfortunately, the neurons representing $\beta$, or any other quantity, do not respond independently of each other. Some covariation in response is inevitable, because any pair of neurons receive a fraction of their inputs in common, a necessity illustrated by Figure 7. The preceding section suggests that the amount of shared input necessary to elicit a small covariation in spike discharge may be quite substantial, but even a small departure from independence turns out to be important. It is easy to see why; any noise that is transmitted via common inputs cannot be averaged away. This is true even when the number of inputs is very large. For example, Zohary et al. (1994) showed that the signal-to-noise ratio of the averaged response cannot exceed $\bar{r}^{-1 / 2}$ where $\bar{r}$ is the average correlation coefficient among pairs of neurons.

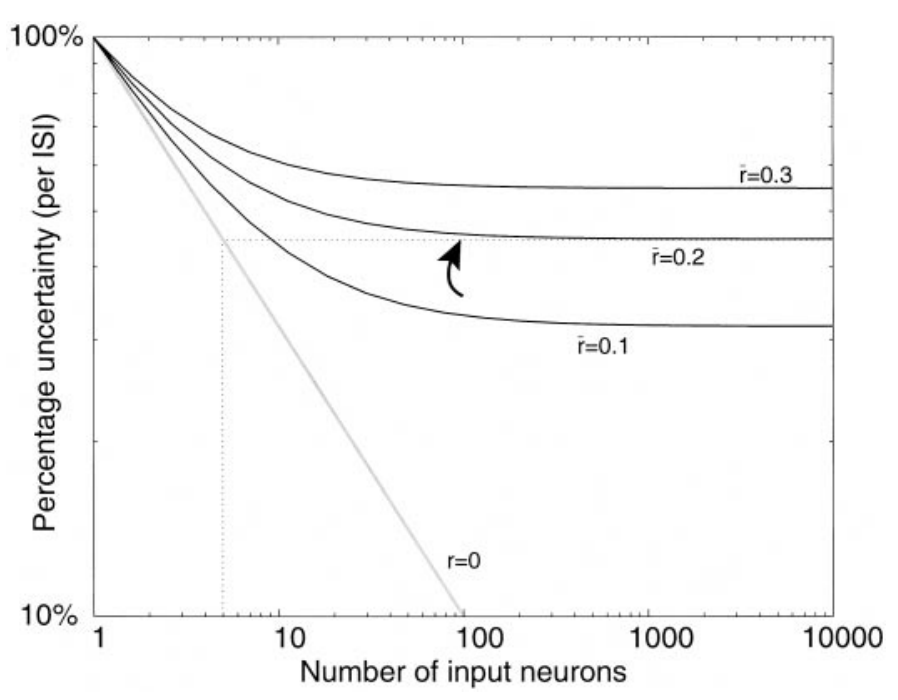

Figure 10. Weak correlation limits the fidelity of a neural code. The plot shows the variability in the number of spikes that arrive in an average ISI from a pool of input neurons modeled as Poisson point processes. Pool size is varied along the abscissa. In one ISI, the expected number of input spikes equals the number of neurons. Uncertainty is the SD of the input spike count divided by the mean. For one input neuron, the uncertainty is $100 \%$. The diagonal gray line shows the expected relationship for independent Poisson inputs; uncertainty is reduced by the square root of the number of neurons. If the input neurons are weakly correlated, then uncertainty approaches an asymptote of $\sqrt{\bar{r}}$ (see Appendix 2). For an average correlation of 0.2 , the uncertainty from a pool of 100 neurons (arrow) is approximately the same as for five independent neurons or, equivalently, the count from one neuron in an epoch of five average ISIs.

We would like to know how correlation among input neurons affects the variability of neural responses at the next level of processing. We can start by asking how variable are the quantities that a neuron inherits to incorporate in its own computation. From the perspective of one of the neurons in the top tier of Figure $7 B$, what is the variability in the number of spikes that it receives from neurons in the middle layer? In other words, how unreliable is the estimate of $\beta$ ?

The answer is shown in Figure 10. We have calculated the uncertainty in the number of spikes arriving from an ensemble of neurons in the middle layer. Each curve in Figure 10 shows uncertainty as a function of the number of neurons in the input ensemble, where uncertainty is expressed as the percentage variation ( $\mathrm{SD} / \mathrm{mean})$ in the number of spikes that a neuron in the top layer would receive from the middle layer in an epoch lasting one typical ISI. This characterization of variability is appealing, because it bears directly on neural computation at a natural time scale.

If there is just one neuron, then the mean number of spikes arriving in an average ISI is one, of course, and so is the SD, assuming a Poisson spike train. Hence the uncertainty is $100 \%$. If there are 100 inputs from the middle layer, then the expected number of spikes is 100: one spike per neuron. If each spike train is an independent Poisson process (Fig. 10, gray line), then the SD is 10 spikes ( 0.1 spikes per neuron), for a percentage uncertainty of $10 \%$. If the spike trains are weakly correlated, however, then the percentage uncertainty is given by:

$$
\% \text { variation }=100 \sqrt{\frac{1+m \bar{r}-\bar{r}}{m}},
$$


where $m$ is the number of neurons, and $\bar{r}$ is the average correlation coefficient among all pairs of input neurons (see Appendix 2). Each of the curves in Figure 10 was calculated using a different value for $\bar{r}$. The solid curves indicate the approximate level of correlation that is believed to be present among pairs of cortical neurons and that is consistent with our simulations using a large fraction of common input $(\bar{r}=0.1-0.3)$. Even at the lower end of this range, there is a substantial amount of variability that cannot be averaged away. For an average correlation coefficient of 0.2 , the percentage uncertainty for 100 neurons is $45 \%$; only a twofold improvement (approximately) over a single neuron!

Three important points follow from this analysis. First, modest amounts of correlated noise will indeed lead to substantial uncertainty in the quantity $\beta$, received by the top tier neurons in Figure $7 B$ that compute $\gamma$, even if $\geq 100$ neurons provide the ensemble input. This variability in the input quantity will influence the variance of the responses of the top tier neurons, an issue to which we shall return in section 3 . Second, the modest reduction in uncertainty achieved by pooling hardly seems worth the trouble until one recalls that what is gained by this strategy is the capacity to transmit information quickly. For example, using 100 neurons with an average correlation of 0.19 , the brain transmits information in one ISI with the same fidelity as would be achieved from one neuron for five times this duration. This fact is shown by the dotted lines in Fig. 10. If we interpret the abscissa for the $r=0$ curve as $m$ ISIs from one neuron (instead of one ISI from $m$ input neurons), we can appreciate that the uncertainty reduction achieved in five ISIs is approximately the same as the uncertainty achieved by about 100 weakly correlated neurons $(\bar{r}=$ 0.2; Fig. 10, arrow). Third, the fidelity of signal transmission approaches an asymptote at 50-100 input neurons; there is little to be gained by adding more inputs. This observation holds for any realistic amount of correlation, suggesting that 50-100 neurons might constitute a minimal signaling unit in cortex. Here lies an important design principle for neocortical circuits. Returning to Figure 7, we can appreciate that the more neurons that are used to transmit a signal, the more common inputs the brain is likely to use. The strategy pays off until an asymptotic limit in speed and accuracy is approached: $\sim 50-100$ neurons.

A most surprising finding of sensory neurophysiology in recent years is that single neurons in visual cortex can encode nearthreshold stimuli with a fidelity that approximates the psychophysical fidelity of the entire organism (Parker and Hawken, 1985; Hawken and Parker, 1990; Britten et al., 1992; Celebrini and Newsome, 1994; Shadlen et al., 1996). This finding is understandable, however, in light of Equation 3, which implies that psychophysical sensitivity can exceed neural sensitivity by little more than a factor of 2 , given a modest amount of correlation in the pool of sensory neurons.

\section{4: Synchrony among input neurons}

If pairs of neurons carrying similar signals are indeed correlated, it is natural to inquire whether such correlation influences the spiking interval statistics considered earlier. How do synchronous spikes such as those reflected in the cross-correlograms of Figure 8 influence the postsynaptic neuron? What is the effect on ISI variability of weak correlation and synchronization in the input spike trains themselves (recall that the inputs in the simulations of Fig. 8 were independent)?

We tested this by simulating the response of two neurons using inputs with pairwise correlation that resembles Figure 8E. We generated a large pool of spike trains using our counting model

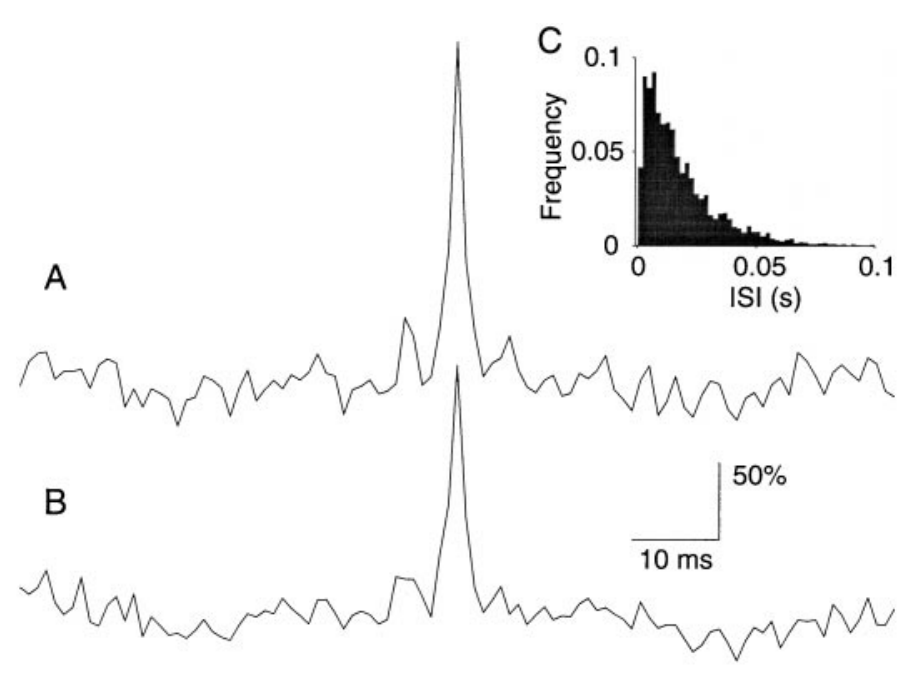

Figure 11. Homogeneity of synchrony among input and output ensembles of neurons. $A$, Normalized cross-correlogram from a pair of neurons receiving 300 excitatory and inhibitory inputs, the typical pairwise crosscorrelogram of which is shown in $B$. The pair share $40 \%$ common excitatory and inhibitory input. The CCG was computed from $801 \mathrm{sec}$ epochs. The simulation produced a correlation coefficient of 0.29 . $B$, The average correlogram for pairs of neurons serving as input to the pair of neurons, whose CCG is shown in $A$. The correlogram was obtained from $801 \mathrm{sec}$ epochs using randomly selected pairs of input neurons. The mean correlation coefficient, $\bar{r}$, was 0.3 . Vertical scale reflects percent change in the odds of a spike, relation to background. $C$, Spike interval histogram for the output neurons. Synchrony among input neurons does not lead to detectable structure in the output spike trains $\left(C_{\mathrm{V}_{\mathrm{ISI}}}=0.94\right)$.

with 300 excitatory and 300 inhibitory inputs. Each spike train was generated by drawing 300 inputs from a common pool of 750 independent Poisson spike trains representing excitation and another 300 inputs from a common pool of 750 Poisson spike trains, which represented the inhibitory input. The strategy ensures that, on average, any pair of spike trains was produced using $40 \%$ common excitatory input and $40 \%$ common inhibitory input. Thus any pair of spike trains has an expected correlation of 0.25-0.3 and a correlogram like the one in Figure 8E. We simulated several thousand responses in this fashion and used these as the input spike trains for a second round of simulations. The correlated spike trains now served as inputs to a pair of neurons using the identical model. Again, $40 \%$ of the inputs to the pair were identical. By using the responses from the first round of simulations as input to the second, we introduced numerous synchronized spikes to the input ensemble.

The result is summarized in Figure 11. The cross-correlogram among the output neurons (Fig. 11A) resembles the correlogram obtained from the inputs (Fig. $11 B$ ). We failed to detect an increase in synchrony. In fact the correlation coefficient among the pair of outputs was 0.29 , compared with 0.30 for the inputs. The synchronized spikes among the input ensemble did not lead to more synchronized spikes in the two output neurons. Nor did input correlation boost the spike rate or cause any detectable change in the pattern of spiking. As in our earlier simulations, the output response was approximately the same as any one of the 600 inputs. Moreover, the spike trains were highly irregular, the distribution of ISIs approximating an exponential probability density function (Fig. 11, inset; $C_{\mathrm{V}_{\mathrm{ISI}}}=0.94$ ). We detected no structure in the output response or in the unit autocorrelation functions.

The finding contradicts the common assumption that synchro- 
nous spikes must exert an exaggerated influence on networks of neurons (Abeles, 1991; Singer, 1994; Aertsen et al., 1996; Lumer et al., 1997). This idea only holds practically when input is relatively sparse so that a few presynaptic inputs are likely to yield a postsynaptic spike (Abeles, 1982; Kenyon et al., 1990; Murthy and Fetz, 1994). The key insight here is that the cortical neuron is operating in a high-input regime in which the majority of inputs are effectively synchronous. Given the number of input spikes that arrive within a membrane time constant, there is little that distinguishes the synchronous spikes that arise through common input. If as few as $5 \%$ of the $\sim 3000$ inputs to a neuron are active at an average rate of 50 spikes/sec, the neuron receives an average of 75 input spikes every $10 \mathrm{msec}$. The random walk mechanism effectively buffers the neuron from the detailed rhythms of the input spike trains just as it allows the neuron to discharge in a graded fashion over a limited dynamic range.

\section{3: Noise propagation and neural computation}

As indicated previously, a remarkable property of the neocortex is that neurons display similar statistical variation in their spike discharge at many levels of processing. For example, throughout the primary and extrastriate visual cortex, neurons exhibit comparable irregularity of their ISIs and spike count variability. When an identical visual stimulus is presented for several repetitions, the variance of the neural spike count has been found to exceed the mean spike count by a factor of $\sim 1-1.5$ wherever it has been measured (see Background). The apparent consistency implies that neurons receive noisy synaptic input, but they neither compound this noise nor average it away. Some balancing equilibrium is at play.

Recall that our simulations led to a spike count variance that was considerably less than the mean count (Fig. 6), in striking contrast to real cortical neurons. Because the variance of the spike count affects signal reliability, it is important to gain some understanding of this fundamental property of the response. In this section we will develop a framework for understanding the relationship between the mean response and its variance under experimental conditions involving repetitions of nominally identical stimuli. Why does the variance exceed the mean spike count, and how is the ratio of variance to mean count preserved across levels of processing? The elements of the variance turn out to be the very quantities we have enumerated in the preceding sections: irregular ISIs and weak correlation.

\section{1: Background and terminology}

At first glance it may seem odd that investigators have measured the variance of the spike count; after all it is the $S D$ that bears on the fidelity of the neural discharge. However, a linear relationship between the mean count and its variance would be expected for a family of stochastic point processes known as renewal processes. A stochastic point process is a random sequence of stereotyped events (e.g., spikes) that are only distinguishable on the basis of their time of occurrence. In a renewal process the intervals from one event to the next (e.g., ISIs) are independent of one another and drawn from a common distribution (i.e., they are independent and identically distributed; Karlin and Taylor, 1975). The Poisson process (e.g., radioactive decay) is a well known example. Recall that the intervals of a Poisson process are described by the exponential probability density function:

$$
f(x)=\lambda e^{-\lambda x}
$$

where $\lambda$ is the average event rate, and $\lambda^{-1}$ is both the mean interval and SD (i.e., $C_{\mathrm{V}}=1$ ). The number of events observed in an epoch of duration, $T$, is also a random number. The count, which we shall denote, $N(T)$, obeys a Poisson distribution; the mean count is $\lambda T$, as is the variance. We will use angled brackets to indicate the mean of many repetitions and summarize the Poisson case by writing, $\operatorname{Var}[N(T)]=\langle N(T)\rangle=\lambda T$. For any renewal process (not just the Poisson process), the number of events counted in an epoch, $N(T)$, is a random number with variance that scales linearly with the mean count. The constant of proportionality is the squared coefficient of variation $\left(C_{\mathrm{V}}\right)$ of the interval distribution: $\operatorname{Var}[N(T)]=C_{\mathrm{V}}^{2}\langle N(T)\rangle$ (Smith, 1959).

Spike trains from cortical neurons bear certain similarities to Poisson processes, and it is presumably for this reason that investigators have sought a lawful relationship between the mean spike count and its variance. However, as we have noted, the spike counts from cortical neurons exhibit even greater variance than the Poisson case: $\operatorname{Var}[N(T)]\rangle\langle N(T)\rangle$. In general, real spike trains are not renewal processes. ISIs often fail independence (e.g., during bursts), and, unless the spike rate is constant, ISIs cannot be described by a common distribution (Teich et al., 1997). The latter concern is particularly important when we consider the behavior of the neuron over many repetitions, for as we will see in a moment, the spike rate is emphatically not the same from epoch to epoch. In contrast to real spike trains, the random walk model described in section 1 produced a sequence of independent ISIs, described by a common distribution, whether viewed in one or several epochs; it describes a renewal process. We would like to relate this process to the variance of the spike count that would be observed over many stimulus repetitions.

Table 2 lists the main mathematical symbols used in our argument as well as some guidance to their interpretation. The scenario we will develop can be summarized as follows. In an epoch of duration, $T$, the neuron receives $n_{\mathrm{i}}(T)$ synaptic inputs from $m$ input neurons $(i=1 . . m)$. The postsynaptic neuron computes some quantity from these inputs, which it attempts to represent as a spike rate, $\lambda$, by emitting a sequence of spikes modeled as a renewal. From here on we will no longer simulate spike trains using the random walk model and instead identify a desired spike rate - the result of some neural computation-and adopt $\lambda^{-1}$ as the expected ISI of a renewal process. The strategy will allow us to write equations for the spike count variance.

\section{2: Two components of response variance}

The observed spike count variance can be divided into two components. The first is related to the irregular ISIs that would arise from a renewal process when the rate is known. We will refer to this source of variance as conditional noise, because it assumes precise knowledge of the desired output spike rate. It is the variance in the spike count that we would anticipate if a neuron were to compute the same quantity on each stimulus repetition. It is the kind of variability that we might associate with a Poisson process (or some other renewal) when the rate is known. The second source of noise reflects the fact that for any one spike train, we do not really know the expected rate with certainty. For repeated presentations of the same stimulus, the quantity actually computed by the neuron varies from trial to trial. As we discovered in section 2, this is because the inputs are weakly correlated. This source of variability might be called extrinsic noise or, more accurately, the variability of the computed expectation.

In general, when a random quantity, $y$, depends on a second quantity, $x$, which is also variable, the variance of $y$ can be expressed as the sum: 


\begin{tabular}{|c|c|c|}
\hline Symbol & Interpretation & Notes \\
\hline$T$ & Duration of epoch & Chosen by experimenter \\
\hline$N(T)$ & Number of spikes from the output neuron in epoch, $T$ & Measured experimentally \\
\hline$\langle N(T)\rangle$ & $\begin{array}{l}\text { Mean spike count from the output neuron; also read } \\
\text { as the expectation of the spike count }\end{array}$ & Measured experimentally using repetitions of identical stimuli \\
\hline $\operatorname{Var}[N(T)]$ & Variance of spike count from the output neuron & Measured experimentally using repetitions of identical stimuli \\
\hline$n_{\mathrm{i}}(T)$ & $\begin{array}{l}\text { Number of spikes from the } i^{\text {th }} \text { input neuron in epoch, } \\
\quad T\end{array}$ & $\begin{array}{l}\text { Unknown; we assume that input and output spike counts are the } \\
\text { same, on average: }\langle N(T)\rangle=\left\langle n_{\mathrm{i}}(T)\right\rangle\end{array}$ \\
\hline$\lambda$ & $\begin{array}{l}\text { Intended spike rate of the output neuron; result of the } \\
\text { neural computation }\end{array}$ & $\begin{array}{l}\text { Theoretical quantity; assumed to be constant over the epoch, so } \\
\langle N(T)\rangle=\langle\lambda T\rangle \text {; this assumption can be relaxed, however; then, } \\
\langle N(T)\rangle=\left\langle\int^{T} \lambda(t) d t\right\rangle\end{array}$ \\
\hline$C_{\mathrm{V}_{\mathrm{ISI}}}$ & $\begin{array}{l}\text { Coefficient of variation of ISI distribution, which char- } \\
\text { acterizes a renewal process: } \sigma_{\text {ISI }} / \mu_{\text {ISI }}\end{array}$ & $\begin{array}{l}\text { Theoretical quantity; can only be measured when } \lambda \text { is constant; } \\
\text { for the renewal processes considered here } C_{\mathrm{V}_{\mathrm{ISI}}} \text { is the same for } \\
\text { all possible values of } \lambda\end{array}$ \\
\hline $\bar{r}$ & $\begin{array}{l}\text { Average correlation coefficient between pairs of input } \\
\text { neurons }\end{array}$ & $\begin{array}{l}\text { Estimated experimentally from pairwise recording, using repeti- } \\
\text { tions of identical stimuli }\end{array}$ \\
\hline
\end{tabular}

Note the distinction between experimentally measurable and theoretical quantities.

$$
\operatorname{Var}[y]=\langle\operatorname{Var}[y \mid x]\rangle+\operatorname{Var}[\langle y \mid x\rangle],
$$

where $\langle\cdots\rangle$ denotes the average over all values of $x$. The expression $y \mid x$ denotes the random value, $y$, conditional on knowledge that $x$ has taken on some particular value. In words, Equation 5 says that the observed variance of $y$ is the sum of its mean conditional variance plus the variance of its conditional mean. The equation applies intuitively to random numbers drawn from a distribution with mean and variance that change on every pick. The conditional variance describes the variance that would apply on any one draw, whereas the variance of conditional mean describes the distribution of expectations across the picks.

In a similar fashion, we can conceive of the spike count as a random number drawn from a distribution with expectation that changes for each repetition of the stimulus or trial. The expectation reflects some computation on the input spike trains. Thus on any one trial, the neuron computes a spike rate, $\lambda$, and emits a random spike train with an expected count of $\lambda T$. Representing the spike count by $N(T)$, we can rewrite Equation 5 as follows:

$$
\begin{aligned}
\operatorname{Var}[N(T)] & =\langle\operatorname{Var}[N(T) \mid \lambda]\rangle+\operatorname{Var}[\langle N(T) \mid \lambda\rangle] \\
& =\langle\operatorname{Var}[N(T) \mid \lambda]\rangle+\operatorname{Var}[\langle\lambda T\rangle] .
\end{aligned}
$$

The idea is that the variance of the spike count can be divided into a portion that would be present even if the neuron were to compute the same value for $\lambda$ on every trial and another portion that reflects the fact that it does not compute the same $\lambda$ on every trial. $^{b}$

It is helpful to relate this concept briefly to the counting model used in previous sections. In section 1, we designed the model to respond at a spike rate equal to the average of its inputs. For $m$ inputs, the computation is:

\footnotetext{
$\overline{{ }^{b} \text { Knowledge of } \lambda \text { does not imply exact knowledge of the input spike trains. Presum- }}$ ably there are many patterns of inputs that give rise to the same result, $\lambda$. Because our model for synaptic integration is deterministic, identical inputs would produce identical outputs. If $\mathbf{x}$ were an exhaustive description of the input spike trains, that is, the time of every spike among all excitatory and inhibitory inputs, then $\operatorname{var}[N(T) \mid \mathbf{x}]=0$. To make sense of Equation 5, we need to make it clear that what we know about the inputs, as reflected in the conditional probabilities, is a scalar value that is computed from them, i.e., $\lambda$.
}

$$
\lambda=\frac{1}{m T} \sum_{i=1}^{m} n_{\mathrm{i}}(T),
$$

where $n_{\mathrm{i}}(T)$ is the input spike count from the $i^{\text {th }}$ input neuron during the epoch $T$. Thus, $\lambda$ reflects the average spike rate among the active inputs, and the computation satisfies our desire for homogeneity of input and output spike rates. Recall from section 1 that the model produced a variable spike count even though the average input rate was the same on each repetition. Because the inputs were modeled as independent, there was little variation in the mean rate of the inputs from trial to trial. Hence $\lambda$ was known precisely, or nearly so, implying that the variance of the conditional mean was negligible. In short, all of the spike count variance is attributed to the renewal process. When correlation was introduced among the inputs, however (section 2), additional variance arose because of the uncertainty in $\lambda$. Equation 6 tells us that these two sources of variance add.

Figure 12 is intended to convey an intuition for the two sources of variance and their importance for networks of interconnected neurons. The three neurons at the top of Figure 12 are idealized as Poisson generators, and all compute the same quantity, $\lambda$, which is the mean input spike rate (Eq. 7). The neurons at the bottom provide the input spikes; they are also Poisson generators and represent the same expected rate. (By Poisson generator, we mean that the neuron computes a rate value and generates a spike train with intervals that are independent and exponentially distributed.) Suppose that the input spike rate is 100 spikes/sec and $T=100 \mathrm{msec}$. Then the expected spike count from the input neurons is $\left\langle n_{\mathrm{i}}(T)\right\rangle=10$ spikes, and the variance is 10 spikes $^{2}$, as shown by distribution in the bottom graph. Each of the neurons shown at the top of Figure 12 computes the average spike rate among the inputs: 100 spikes/sec, or 10 spikes in the $100 \mathrm{msec}$ epoch. The top graphs depict the distribution of spike counts emitted by these "output" neurons.

If there were just one input neuron, as in Figure 12A, then two sources of variance, extrinsic and conditional in the terms established above, would combine to produce an output that is more variable than the single neuron input. Extrinsic noise reflects the fact that the input is not constant on each trial but instead takes 


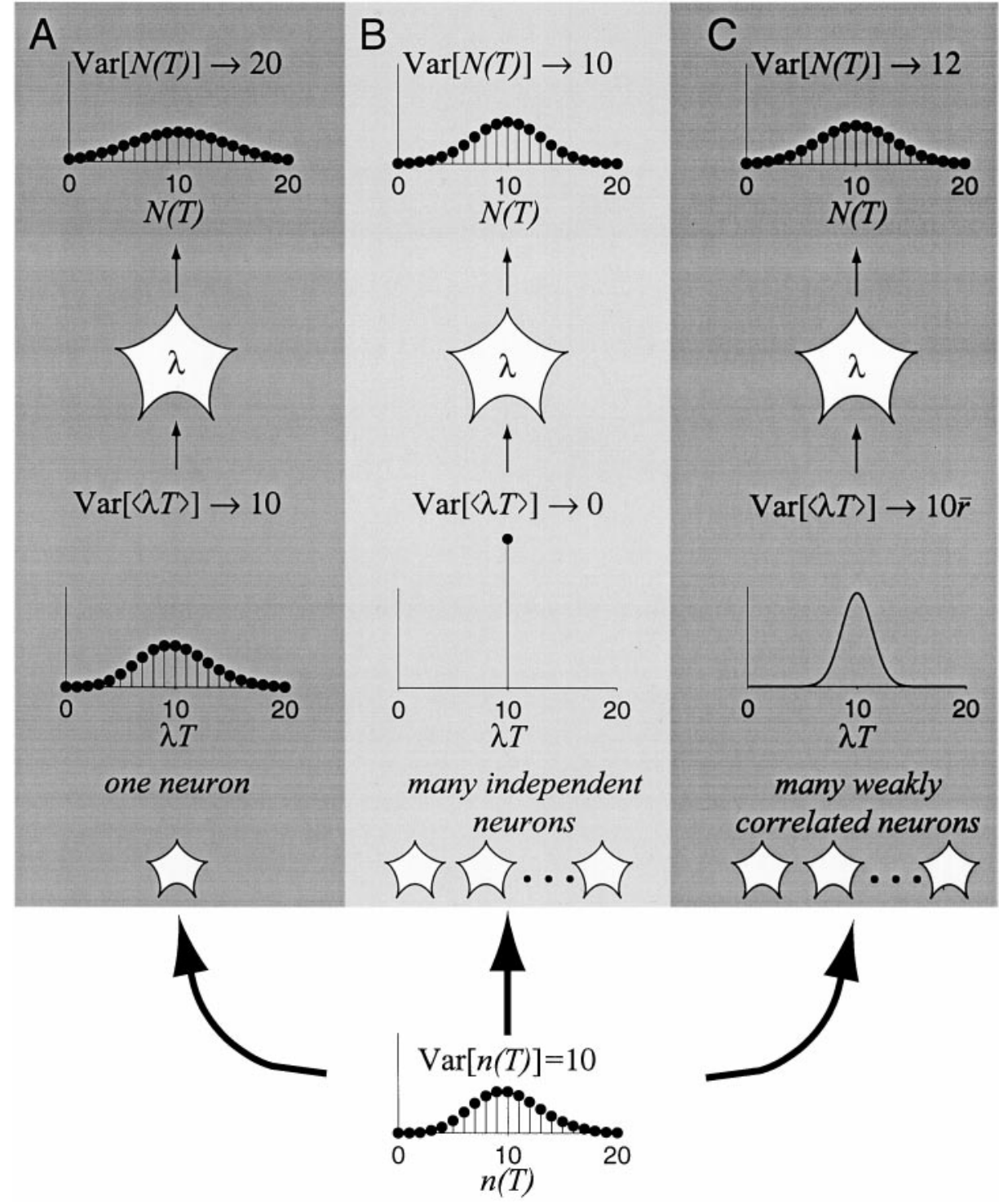

Figure 12. Propagation of noise among ensembles of neurons. Each of the neurons depicted in this figure emits a spike train idealized as a Poisson point process. The three neurons at the top compute the average spike rate among their inputs and emit the answer as a Poisson spike train. During a $100 \mathrm{msec}$ epoch, the input neurons (bottom) discharge at 100 spikes/sec. Each input neuron is therefore expected to generate 10 spikes, but in any one epoch the count may vary. The bottom graph shows the Poisson distribution of spike counts from one input neuron. The middle row of graphs shows the probability density of the quantity that the output neuron has computed: the number of spikes per input neuron. The neuron obtains an estimate of the input spike count by calculating with one $(A)$ or more neurons $(B, C)$. The value is represented as an expectation, $\langle\lambda T\rangle$, which can be thought of as a desired rate times the epoch duration. In any one epoch, the neuron emits a Poisson spike train at a rate, $\lambda$, resulting in $N(T)$ spikes. The distribution of $N(T)$ from many $100 \mathrm{msec}$ repetitions is shown at the top. $A$, If the output neuron receives input from just one input neuron, the variance of the input count, $\operatorname{var}[\langle\lambda T\rangle]$, is 10 . The output neuron emits an average of 10 spikes, but the variance is 20 , reflecting the sum of input and (its own) Poisson variance. $B$, If there are many independent inputs, then the variance of the mean input count is negligible (delta function; middle plot). The output neuron emits an average of 10 spikes, and the variance is 10 , the amount of variance expected for a Poisson spike train. $C$, If there are many weakly correlated input neurons, then the variance of the mean input count is approximately 10 times the average correlation coefficient among the input neurons. If $\bar{r}=0.2$, then the variance is 2 . The output neuron emits an average of 10 spikes, but the total variance is 12 . Notice that in $A$ and $C$, the variance of the output spike count exceeds the variance of the inputs. the form of a random pick from the probability distribution shown in the middle row. This is the distribution of $\lambda T$ estimated by applying Equation 7 to just one neuron. Thus, we imagine that the output neuron receives a random pick of $\lambda T$ from this distribution and generates a Poisson spike train with this expected count. The total variance is the sum of the extrinsic (input) variance and the conditional variance arising from the Poisson renewal process. In this case the mean is 10 spikes, and the variance is 20 spikes $^{2}$. In just one synapse the variance-to-mean ratio has doubled. Such a scheme is obviously untenable for extended chains of neural processing.

If the neuron were to compute the average spike rate from many independent neurons, then the extrinsic noise would approach zero. This is represented by the delta function in Figure $12 B$ (middle row). On every repetition, the computation produces 100 spikes/sec (10 spikes/100 msec per neuron) by pooling across the ensemble of input neurons. The output neuron necessarily incorporates conditional noise and therefore generates a Poisson spike train with an expected count of 10 and a variance of 10 . The output response is only as variable as a Poisson process with rate 100 spikes/sec. This situation resembles the simulations per- formed in section 1.4. The variance of the mean input count was negligible because we simulated each of the inputs independently. Here we have simply bypassed the random walk model and instead postulated that the neuron computes $\lambda=100$ spikes/sec and emits a Poisson spike train accordingly.

A more realistic situation, involving correlated noise among the inputs, is shown in Figure 12C. Again, we suppose that the neuron computes the mean of many input spike counts, but that the response of any one input neuron is correlated weakly with the response of the others. Averaging no longer leads to a precise value but retains some variability. For the computation in Equation 7 , the variance would be estimated by multiplying the average correlation coefficient, $\bar{r}$, by the variance of a single input:

$$
\operatorname{Var}[\langle\lambda T\rangle] \approx \bar{r} \operatorname{Var}[n(T)] .
$$

This approximation is implied by Equation 3 for large numbers of inputs (see Appendix 2). If $\bar{r}=0.2$, then the variance of the computed quantity would be 2 spikes $^{2}$. The value transmitted to the neuron at the top of Figure 12 is 10 spikes per neuron per epoch, with a variance of 2 . Once again, the top neuron repre- 
sents this quantity by emitting a Poisson spike train. The total variance in this case is 12 spikes $^{2}, 1.2$ times the mean.

The idea that spike count variance arises as a sum of conditional and input variances is appealing for its simplicity. It provides some intuition about how the spike count variance would exceed its mean, and it suggests that the ratio of spike count variance to the mean count ought to be a constant. However, we have yet to achieve an adequate explanation for the apparent homogeneity of this relationship among input and output neurons. The example in Figure $12 B$ would be regarded as successful were it not for the unrealistic assumption of independent inputs, hence noise-free computation. The example in Figure $12 C$ comes close, but the variance of the input neurons at the bottom does not match the variance of the spike count produced by the neuron at the top (10 and 12, respectively). It would be untenable for neurons to boost the variance by $20 \%$ at each stage of synaptic integration.

\section{3: A stable solution for spike count variance}

We therefore seek a stable ratio of variance to mean spike count, one that encompasses input neurons and output neurons alike and one that more accurately reflects the variability of real neurons. For the moment, we will continue to make our simplifying assumption that the neuron computes the average firing frequency of its active inputs (Eq. 7). This case allows us to elaborate the scheme depicted in Figure 12 and serves as a reference point for further discussion.

In section 1.2 we established that a random walk model for synaptic integration allows the neuron to respond at a rate that is approximately equal to the average rate of its inputs, but that the output spike train is highly irregular. Recall that the coefficient of variation of the ISIs $\left(C_{\mathrm{V}_{\mathrm{ISI}}}\right)$ was $0.8-0.9$ for a variety of model parameters. When we computed the spike counts from many epochs, we found that the variance-to-mean ratio of the spike counts was 0.75 on average (Fig. 6). This value is sensible because the random walk model produced spike trains that conformed to a renewal process: spike intervals were independent, and the expected spike rate was the same for all repetitions. As noted earlier, the predicted relationship between the spike interval distribution and the variance in the number of spikes counted in an epoch, $T$, is given by $\operatorname{Var}[N(T)]=C_{\mathrm{V}_{\text {ISI }}}^{2}\langle N(T)\rangle$, which would predict a variance-to-mean ratio of $0.64-0.81$. This relationship presumes a fixed spike rate, $\lambda$. If $\lambda$ is variable, however, this same relationship then applies only to the average conditional variance:

$$
\langle\operatorname{Var}[N(T) \mid \lambda]\rangle \approx\left\langle C_{\mathrm{V}_{\mathrm{ISI}}}^{2} N(T) \mid \lambda\right\rangle=C_{\mathrm{V}_{\mathrm{ISI}}}^{2}\langle N(T)\rangle .
$$

Equation 9 works because the $C_{\mathrm{V}_{\mathrm{ISI}}}$ is the same regardless of spike rate. $^{c}$ The left side of Equation 9 is the average of the variances computed at each fixed spike rate. We referred to this as the mean conditional variance in Equation 5. It is the amount of variance that we would observe if only the neuron were instructed to produce the same spike rate in each epoch.

In reality, of course, correlation among the inputs guarantees that the neuron receives somewhat different instructions on each trial. This additional variance is represented by the second term in Equation 6: the variance of the conditional mean. The amount

\footnotetext{
${ }^{c}$ For many stochastic processes, including our random walk model, a change in rate is equivalent to scaling time. Hence the $C_{\mathrm{V}}$ is constant. For these cases, Equation 9 also holds for time varying spike rates, $\lambda(t)$, as long as the rate function can be repeated for each epoch contributing to the conditional variance. Thus, for a nonstationary Poisson process, the variance of the counts equals the mean of the counts.
}

of this additional variation depends on what the neuron is calculating. If the neuron computes the number of spikes per active input, then we can use the expression in Equation 8. The total variance is therefore:

$$
\operatorname{Var}[N(T)]=C_{\mathrm{V}_{\mathrm{ISI}}}^{2}\langle N(T)\rangle+\bar{r} \operatorname{Var}[n(T)] .
$$

This equation is obtained by combining Equations 6-9. It says that the variance of the spike count reflects (1) the irregularity of the spike train, and (2) an inability to average out common noise.

We seek conditions in which the input and output neurons exhibit the same degree of variability. This would describe a steady state in which noise neither compounds nor attenuates in a neural processing chain. For the mean spike rate calculation (Eq. 7), the expected counts are the same for input and output neurons. In the steady state, the expected variance of the input and output neurons must be equal as well: $\operatorname{var}[N(T)]=\operatorname{var}[n(T)]$. This equality allows us to rearrange Equation 10 to yield a steady-state solution:

$$
\operatorname{Var}[N(T)]=\frac{C_{\mathrm{V}_{\mathrm{ISI}}}^{2}}{1-\bar{r}}\langle N(T)\rangle .
$$

This expression tells us the relationship between the variance and the mean spike count for any neuron in any tier of a network that is designed to compute the mean response of its inputs.

In Figure 12, we assumed that the neuron behaves as a Poisson generator. Choosing $C_{\mathrm{V}_{\mathrm{ISI}}}=1$ and $\bar{r}=0.2$, Equation 11 indicates that the variance of the spike count should be 1.25 times its mean value. This is the homogeneous solution for populations of neurons that behave as Poisson generators whose spike rate is the mean of the input spike rates. If the input neurons in Figure $12 \mathrm{C}$ had a spike count variance of 12.5 (instead of 10), then the variance of the output, shown at the top, would have been 12.5 as well. The neuron at the top of Figure $12 C$ would inherit a variance of 2.5 (because $\operatorname{Var}[\langle\lambda T\rangle] \approx \bar{r} \operatorname{Var}[n(T)]=(0.2)(12.5)=2.5)$, which adds to the conditional variance of the Poisson generator $\left(10\right.$ spikes $\left.^{2}\right)$. The simulations in section 1 lead us to suggest that a better estimate of the $C_{\mathrm{V}_{\mathrm{ISI}}}$ might be 0.8 (Figs. 4 and 5). The stable solution for the variance is then 0.8 times the mean (by Eq. 11 ), which is less than the value of 1-1.5, measured experimentally.

It is possible that this discrepancy between theory and experiment arises from additional sources of signal variability in vivo, such as fluctuations in eye position in awake animals (Gur et al., 1997) or fluctuations in cortical excitability (Arieli et al., 1996). More likely, in our opinion, is that our theoretical analysis underestimates the real variance, because we have assumed a very simple computation-averaging. In the next section we will explore this proposition.

\section{4: Neural calculations other than the mean}

Throughout this paper we have assumed that a cortical neuron computes something like the average rate of its inputs. This was a convenient choice to satisfy the homogeneity constraints listed in Table 1. In general, however, a postsynaptic neuron is likely to compute something more complicated than the average of its inputs, and the variance of this quantity will typically exceed the amount deduced for averaging (Eq. 8). Even to compute the average number of spikes per active input, the neuron needs an estimate of the number of active inputs ( $m$ in Eq. 7). We have tacitly assumed knowledge of this value in Equation 7 and in the adjustment of barrier height in the random walk model. The 
neuron needs an estimate of this quantity, however, and to the extent that this estimate carries uncertainty, it will augment the variance associated with computation $\operatorname{Var}[\langle\lambda T\rangle]$. This is just one of many possible sources of uncertainty that would boost the variance of the neural discharge. We have referred to this additional variance as pooling noise in previous work (Shadlen et al., 1996).

How much additional variance can be anticipated if the neural computation combines several quantities, each carrying its own uncertainty? The following contrivance offers surprising insight. Suppose that in addition to computing the average rate among some set of inputs, the neuron were to add and subtract two other quantities. Let the expected spike rate reflect the addition of two values and the subtraction of a third, as in $a+b-c$. Analogous to Equation 7, we can represent the computation as:

$$
\lambda T=\frac{1}{m_{a}} \sum_{i=1}^{m_{a}} n_{\mathrm{i}}(T)+\frac{1}{m_{b}} \sum_{i=1}^{m_{b}} n_{\mathrm{i}}(T)-\frac{1}{m_{c}} \sum_{i=1}^{m_{c}} n_{\mathrm{i}}(T),
$$

where $m_{a}, m_{b}$, and $m_{c}$ are the number of inputs that convey the quantities $a, b$, and $c$. We are not proposing that this is how a neuron might add and subtract quantities; we use this contrived example only to illustrate a computation with more variance than a simple average. The important aspect of Equation 15 is that it combines distinct entities after the averaging steps. Each term therefore introduces variance into the computation of $\lambda T$. Thus, Equation 15 might be interpreted as a special case of a class of computations that would include multiplication and logical comparisons between inputs arriving at distinct parts of the dendrite. This particular calculation allows us to extend the intuitions gained from the preceding section. If we take the average spike rates of all the inputs to be the same, then the expected output rate is the same as any one of the inputs, and we can apply the same strategy as before to find a stable solution for the spike count variance.

Assume that 100 or more inputs represent each of the three quantities, $a, b$, and $c$, and that the neurons that comprise the pools are weakly correlated among themselves. ${ }^{d}$ The extra quantities boost the uncertainty of the computation. Using the same notation as before, the variance of the conditional expectation reflects the sum of the variances from each of the three terms:

$$
\operatorname{Var}[\langle\lambda T\rangle] \approx 3 \bar{r} \operatorname{Var}[n(T)],
$$

triple the amount in Equation 8. The rest of the argument is identical to the last section. The observed spike count 18 variance reflects this uncertainty plus the variability that arises because of the stochastic nature of the ISI (i.e., the fact that the neuron realizes its intended rate as a renewal process):

$$
\operatorname{Var}[N(T)]=C_{\mathrm{V}_{\mathrm{ISI}}}^{2}\langle N(T)\rangle+3 \bar{r} \operatorname{Var}[n(T)] .
$$

Because the expected spike count is the same for input and output, we can equate the variances to find a stable solution:

$$
\operatorname{Var}[N(T)]=\frac{C_{\mathrm{V}_{\mathrm{ISI}}}^{2}}{1-3 \bar{r}}\langle N(T)\rangle .
$$

Plugging in 0.8 for the $C_{\mathrm{V}_{\mathrm{ISI}}}$ and 0.2 for $\bar{r}$, we obtain a stable variance-to-mean ratio of 1.6 . If input neurons were less variable

\footnotetext{
${ }^{d}$ Weak correlation between the pools representing $a, b$, and $c$ would not affect the calculations substantially, because the resulting covariance terms would offset each other due to the sum and difference.
}
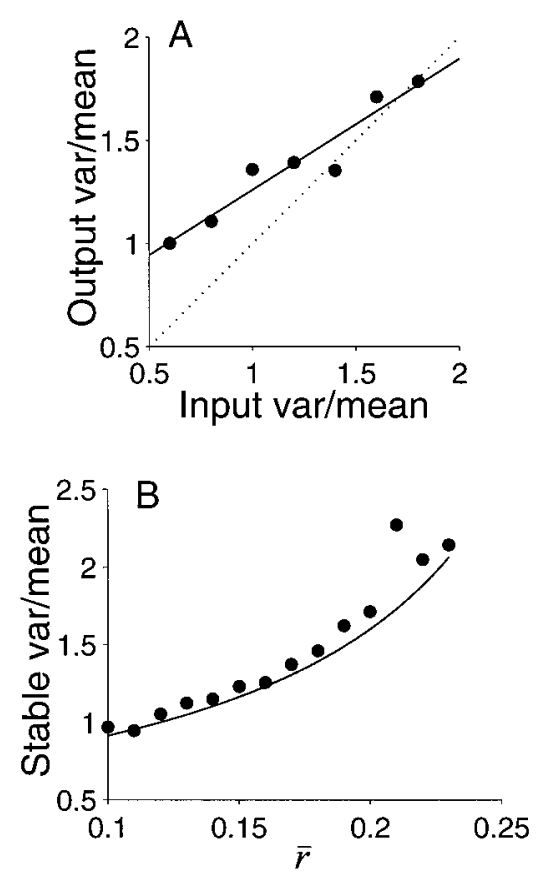

Figure 13. Stable solution for the variance-to-mean spike count in networks of neurons when the computation involves three independent quantities. $A$, Numerical approximation to the stable solution. We simulated the response by estimating three values using 100 weakly correlated neurons per value $(\bar{r}=0.2)$. The three means were combined as a sum and difference $(a+b-c)$ to generate the expected spike count from the output neuron, the spike train of which was modeled as a renewal process with $C_{\mathrm{V}_{\mathrm{ISI}}}=0.8$. The variance-to-mean ratio for the input neurons is shown along the abscissa. The output variance-to-mean ratio is plotted along the ordinate. The least squares fit line crosses the main diagonal at a ratio of 1.6. This variance-to-mean ratio would be common to input and output neurons that compute similarly complex quantities. $B$, Effect of correlation on the stable variance-to-mean ratio. Each point represents a numerical approximation like the one obtained in $A$. The procedure was repeated for a range of average correlation coefficients. The solid line is the theoretical result (Eq. 18). Simulations led to larger estimates of variance, because the number of neurons is finite.

than this, then a neuron that computes the quantity in Eq. 15 would be more variable than its inputs. If the inputs had a variance exceeding 1.6 times the mean count, then the output response would be less variable than the inputs.

This point is illustrated by the numerical simulation in Figure $13 A$. The variance of the spike count is shown for a neuron that computes the sum and difference described by Equation 15. We used 100 neurons in each of the three pools representing the quantities $a, b$, and $c$, and we assumed an average correlation of 0.2 among all neuronal pairs within the pools. We adjusted the variance of the input neuron spike counts, using a variance-tomean ratio of from 0.6 to 1.8 , as shown on the abscissa. The variance of the output neuron response reflects the uncertainty in the computation of $\lambda T$ and the additional variation attributable to the irregularity of the ISI, which we modeled as a renewal with $C_{\mathrm{V}_{\mathrm{ISI}}}=0.8$. Notice that the input and output neurons share the same variability when the variance-to-mean ratio is 1.6 , as predicted by Equation 15. This is the stable value for the varianceto-mean ratio in a network of neurons that compute quantities that necessitate combining sources of variance, in the manner of the sum and difference in Equation 12. In Figure $13 B$ we show the stable solution for a range of correlation strengths. For $\bar{r}$ between 0.1 and 0.2 , the variance-to-mean ratio approximates the value measured experimentally (Table 1 ). 
It is sobering to realize that such a simple calculation leads to such large variance in the neural response. This observation is particularly ominous for models in which separate computations are performed on major dendritic branches, with the resulting quantities being summed or multiplied at the soma (Koch et al., 1983; Shepherd and Brayton, 1987; Segev, 1992; Mel, 1993). Our results suggest that in terms of response variance, little headroom is available for such algebra to occur within the postsynaptic neuron. How, then, do neurons compute complex quantities such as orientation, disparity, and motion while keeping response variance stable? In thinking about this question, we find it useful to distinguish between computations that are performed within the postsynaptic neuron and computations that are imposed from outside the postsynaptic neuron by virtue of its afferent anatomical connections. Our results suggest that the primary computation performed within most neurons is a relatively simple weighted average of its many inputs (a slight elaboration of Eq. 7 yields a weighted average). On the other hand, sophisticated quantities can emerge from a weighted average, provided that the inputs and their weights are arranged properly. This computational principle lies at the heart of most neural network models; complex quantities are computed by means of a network of intricately connected "units," each of which calculates a weighted average of its inputs (Pouget et al., 1998). This principle is also congenial with recent "normalization" models in which neurons in different visual areas compute in a similar manner (linear summation followed by response normalization), whereas the diverse response properties that characterize different cortical areas emerge from different patterns of input (Heeger et al., 1996; Simoncelli and Heeger, 1998).

From this point of view, the burden of neural computation falls on the pattern of connections to each postsynaptic neuron or, by extension, on the pattern of connections to the cortical column. The present analysis predicts, in other words, that cortically derived properties such as orientation and direction tuning will be apparent in the organization of synaptic inputs to layer 4 of a given cortical column (Ferster et al., 1996). Outside of layer 4, the majority of neurons receive ascending input predominantly from other neurons in the same column and will thus inherit substantially similar response properties. The intrinsic circuitry of the column may amplify and modulate the quantities generated by the novel anatomical convergence onto layer 4 (Douglas et al., 1995; Somers et al., 1995).

\section{DISCUSSION}

We have identified several principles of statistical homogeneity that pertain to networks of neurons operating in a high-input regime. The central idea is that from the point of view of statistics, input and output neurons should be indistinguishable. They respond over the same dynamic range; they exhibit the same irregularity in their spike output patterns; and they manifest the same uncertainty in their spike counts over epochs of a few ISIs. These constraints are satisfied by a simple integrate-and-fire model with decay, assuming balanced excitation and inhibition. If cortical neurons operate in this fashion, then the irregular spike patterns they exhibit ought to be interpreted as noise. This implies that the intervals themselves do not convey meaningful information beyond their expression of spike rate. More specifically, precise arrangements of spikes and constellations of intervals among ensembles of neurons are unlikely to play a role in neural signaling. The idea that neurons encode information through modulation of their spike rate is hardly novel, but we have shown that it takes an ensemble of neurons to do so reliably and, moreover, that the ensemble need not exceed 100 neurons. Because the rate can change quickly, our argument is not inconsistent with the broad concept of temporal coding. Indeed, Figure 1 illustrates responses of a neuron with a rate that varies rapidly with changes in the visual stimulus.

Our arguments presuppose that neurons in neocortex receive a surfeit of excitatory synaptic input. This is likely to be so because the conditions that cause a neuron to discharge also cause some considerable fraction of its inputs to discharge as well. This proposition is difficult to quantify precisely, but it is consistent with optical imaging studies, which suggest that simple visual patterns elicit activity in very large populations of neurons (Grinvald et al., 1994; Das and Gilbert, 1995a), and it conforms to the informal observations of a great many physiologists. Where response properties respect a columnar organization, it can be surmised that neurons within a cylindrical radius of 50-100 $\mu \mathrm{m}$ respond under similar conditions. Such a cylinder accounts for approximately half of the 3000-10,000 excitatory synapses to a neuron (Braitenberg and Schüz, 1991; Douglas et al., 1995). Moreover, many of the inputs from outside this cylinder come via horizontal connections from columns with overlapping response properties as well (Ts'o et al., 1986; Gilbert and Wiesel, 1989; Weliky et al., 1995; Bosking et al., 1997). These rules are best documented for the primate visual cortex, but they are probably valid for other regions of neocortex as well (Braitenberg and Schüz, 1991; Amir et al., 1993; Lund et al., 1993). Thus stimulus conditions that induce one neuron to respond also affect a large number of the neuron's inputs.

As long as the neuron operates in such a high-input regime, excitation needs to be balanced with inhibition to maintain a proper dynamic range of responses. Several considerations suggest that the net excitatory and inhibitory inputs may be approximately balanced. At a membrane potential below spike threshold, for example, outward currents follow weaker electrochemical gradients, but they are longer-lasting than inward currents, and their synaptic contacts are positioned closer to the soma (Beaulieu et al., 1992; Kisvarday et al., 1993). In addition, inhibitory inputs tend to make multiple synapses on a given neuron, ensuring more secure transmission (e.g., fewer failures) (Somogyi et al., 1983b; Thomson et al., 1996). To the extent that excitation of the distal dendrite relies on active (voltage-dependent) dendritic conductances to reach the soma (Amitai et al., 1993; Schwindt and Crill, 1995; Stuart and Sakmann, 1995; Johnston et al., 1996; Schwindt and Crill, 1997a), inhibitory input may have a disproportionately large impact by affecting the gain of this amplification mechanism (Bernander et al., 1994; Hoffman et al., 1997; Schwindt and Crill, 1997b). Finally, powerful evidence favoring such a balance emerges from intracellular recordings performed by Ferster (1986) in simple and complex cells of the visual cortex in the cat (also see Douglas et al., 1991; Nelson et al., 1995; Borg-Graham et al., 1996). Contrary to previous expectations, IPSPs, like EPSPs, were elicited predominantly by a bar of the preferred orientation of the cell. This finding is entirely sensible

${ }^{e}$ One way to appreciate this is to try to reconstruct the spike intervals among a subset of input neurons from the pattern of output spikes. This is obviously impossible with just one output neuron, but it is equally hopeless with an arbitrarily large number of output neurons. The requisite information is encoded in the path of the membrane voltage between spikes, but such information is jettisoned from the spike code. If, in principle, there is no way to reconstruct such intervals, then they cannot encode information, except insofar as they reflect changes in spike rate. 
from the point of view of the counting model; the massive excitatory input characteristic of the high-input regime must be balanced by inhibitory input of similar stimulus selectivity.

In the high-input regime, in which excitation and inhibition are in approximate balance, synaptic integration may be viewed as a counting process resembling a random walk. We emphasize that our conclusions apply only to neurons operating in the high-input regime. In such a setting the neural response reflects the activity of a large ensemble of inputs, and the arrival of any one presynaptic spike has negligible effect on the time of a postsynaptic spike. In some brain structures, of course, the high-input regime does not hold, and a small number of inputs reliably produces an action potential. This appears to be the case for the visual relay neurons in thalamus (Hubel and Wiesel, 1961; Hoffmann et al., 1972; Kaplan and Shapley, 1984) and is paramount in brainstem auditory structures that preserve precise temporal information in their spike trains (Oertel, 1983). Such "privileged" connections may also exist at the initial input stage of the neocortex. For example, individual afferents from the thalamus seem to exert a strong influence on visual cortical neurons (Tanaka, 1983; Reid and Alonso, 1995; Stratford et al., 1996). We suspect, however, that this is true for only a small minority of neocortical connections, and we have ignored them in this paper by adopting the generic perspective that the statistical properties of input and output neurons are grossly indistinguishable.

\section{Simplifications in the counting model}

The counting model we have analyzed incorporates several flawed assumptions, most for the sake of simplicity. For example, the model assumes that all PSPs are of identical magnitude. Obviously, this is an overly simple view; different synapses can vary in strength as a function of their location on the dendritic tree, their history of activation on long time scales as in long-term potentiation and depression, and short time scales as in adaptation. Inputs arriving via stronger synapses might be expected to produce a postsynaptic spike with greater probability, but the importance of this stratagem deserves further scrutiny in the context of the high-input regime under consideration here. Although it is true that synchronous input from just a few strong synapses would produce a spike, such events occur against a setting in which the neuron is inundated with synaptic input. Thus it is likely that such spikes, secure and well timed as they may be, are responsible for only a minority of the output spikes of a neuron.

Interestingly, recent advances in synaptic physiology raise the possibility that variation in synaptic efficacy plays a less pivotal role in shaping the response of the neuron than previously thought. Active conductances along the dendrite can increase the effect at the soma of distal synaptic input, thus ensuring more equal access to the spike-generating region of the neuron for all synaptic currents (Cauller and Connors, 1994; Johnston et al., 1996; Cook and Johnston, 1997). Importantly, increased access to the soma via active dendritic conductances reinforces the central problem motivating our preoccupation with the high-input regimen: all 3000-10,000 synaptic inputs to a neuron can influence the discharge of the cell, regardless of their distance from the soma.

In our model we have ignored conductance changes and active membrane properties. Obviously, synaptic integration is not really a counting process but an electrical one involving dynamic conductance changes. A proper model would incorporate the variety of ion channels, their locations on the dendrite, and the propagation of current through the dendrite to the soma. These models are terribly complicated and are therefore difficult to incorporate into network simulations. One salient example of this type of model, moreover, does not predict an irregular ISI (Softky and Koch, 1993). Douglas et al. (1995) have proposed a conduction-based model that makes explicit account of the network of connections of the neuron, but their model does not deal with the time of synaptic inputs and therefore offers limited insight into the statistics of the neural spike train. Recently, Troyer and Miller (1996) analyzed a realistic model of the layer 5 pyramidal cell using a balance of excitation and inhibition. Their model is especially elegant because the parameters (e.g., conductances) were derived from intracellular recording in the striate cortex of the cat (McCormick et al., 1985). This model produces an irregular ISI and therefore supports our contention that the simplified counting process captures essential characteristics of more realistic neural models. In Appendix 1 we implement a conductance-based model similar to the one used by Troyer and Miller (1996) to determine how it performs in the high-input regime with which we are concerned. The model replicates the main results obtained in this paper with the simpler counting model, although it seems to require a substantially larger synaptic conductance (approximately a factor of eight) than has been measured experimentally (Borg-Graham et al., 1996). In fact, it is very difficult to measure synaptic conductance changes in vivo under conditions leading to the kind of response shown in Figure 1. We suspect that existing physiological studies underestimate the actual conductance, but we also believe that singlecompartment models such as ours tend to overestimate conductance (see Appendix 1). Plainly, substantially more work is needed on both the experimental and theoretical fronts to resolve this discrepancy.

Fortunately, the key insight of the random walk model does not depend critically on biophysical details. It is that the path followed by the membrane voltage from one spike to the next is complex, reflecting a large number of synaptic events. The detailed history of input activity cannot be revealed in the time between output spikes, because there are many possible paths leading to each ISI. Whether it is balanced inhibition or some other mechanism that allows hundreds of synaptic inputs to result in just one spike, the detailed timing relationships of inputs at the dendrites cannot be reconstructed from the output spike discharge. A similar argument has been put forth by van Vreeswijk and Sompolinsky (1996) using an entirely different mathematical framework.

\section{Principles of statistical homogeneity}

We have emphasized the notion that the statistical properties of the neural response are similar for input and output neurons alike. These properties, summarized in Table 1, should be interpreted as loose approximations. Some neurons, of course, respond more strongly than others, and spiking patterns can vary to some extent (McCormick et al., 1985; Gray and McCormick, 1996). Nevertheless, it seems likely that the stimulus conditions that drive a given neuron produce similar changes in an appreciable minority of its inputs and immediate targets. Moreover, the neuron's inputs and its targets probably respond over a similar range of spike rates. This general point of view is central to our analysis, but it is little more than a caricature of a somewhat heterogeneous population of neurons. The problem of excess excitation and the consequences of weak correlation that we have discussed do not depend on an exact sense of homogeneity but on this relaxed sense of a common dynamic range. 
In addition to a common dynamic range, cortical neurons exhibit similar response statistics. Interspike intervals and spike counts obey similar properties for cortical inputs and targets (Softky and Koch, 1993). The balanced excitation-inhibition model presented in section 1 of Results mimics this property of cortical neurons, consistently leading to a $C_{\mathrm{V}_{\mathrm{ISI}}}$ near 0.8 . Interestingly, the model suggests that this degree of variability in the output spike train would result nearly independently of the degree of variability of the input spike trains, as indicated in Figure 5. Thus a network of neurons conforming to our model of synaptic integration will find a steady state near the cited value. This finding might help resolve the puzzle of how relatively regular spike trains from thalamus (Funke and Worgotter, 1995) give rise to such irregular spike trains in cortex.

In section 3 of Results we tried to identify conditions that would allow the variance of the spike count to maintain a fixed relationship to its mean through several stages of processing. This is a critically important property of cortical circuitry, because a quantity computed by neurons at one location in the cortex should not be overwhelmed by an accumulation of noise across subsequent synaptic connections leading to a motor response. Across a wide range of cortical areas, the variance of the neural response appears to remain approximately constant at $\sim 1-1.5$ times the mean count.

We quantified two sources of response variance in cortical neurons: the near-Poisson nature of the synaptic integration process (the random walk model) and response covariance resulting from common input. Together these factors account for a surprisingly large amount of the experimentally observed variance, yielding a variance-to-mean ratio of $\sim 0.8$. This result is quite unexpected, because it leaves little headroom for additional variance that would accompany computations more complex than taking a mean (as in our simulations). For example, a relatively simple operation that incorporates just two additional terms leads to a stable variance-to-mean ratio of 1.6, fully accounting for experimentally observed variability. Disconcertingly, Gur et al. (1997) have recently raised the possibility that experimentally measured variance is in fact exaggerated (but see Bair and O'Keefe, 1998), implying that neural calculations may be simpler even than the three-term operation considered above.

As we have shown, response covariance resulting from common input sacrifices fidelity in the sense of signal/noise, but it must be remembered that this redundancy yields a great benefit: speedy transmission of signals from point to point in the cortex. Input and output neurons can only calculate over the same time scale if there is no accumulation of waiting time for the requisite information to arrive at subsequent stages (Knight, 1972). We have assumed that a natural time scale for computation is on the order of $10 \mathrm{msec}$, which is about half of a membrane time constant, or an average ISI of a neuron with a rate of discharge of 100 spikes/sec. Computation on this time scale is only possible if the neuron receives many samples of the quantities that it needs for its calculations. This convergence, or fan-in, of many inputs imposes a requirement of shared connections and, consequently, limits the fidelity of signals embedded in noise, even after averaging.

Finally, our simulations revealed new insights into the effects of spike synchrony in the high-input regime, insights that may ultimately form a fifth homogeneity principle if confirmed by more extensive measurement and simulations. Succinctly, the fraction of synchronous spikes appears to be conserved from an input ensemble to an output ensemble when a common signal is passed from stage to stage in a processing pathway (Fig. 11). Common input leads to a modest percentage of synchronous spikes in an ensemble of neurons, but the synchronous spikes do not appear to propagate consistently or to establish a consistent pattern of activity among neurons at the next level of processing. We failed to observe any tendency for synchronous spikes to dominate the population response as signals propagate from one stage to the next. Again, this observation follows naturally from the exigencies of the high-input regime. Given the large numbers of input spikes that arrive in a given membrane time constant, each arriving spike is effectively synchronous with some population of inputs, although the composition of the "synchronous" population changes randomly from epoch to epoch. Thus synchrony is ubiquitous in the high-input regime; "special" spikes defined by consistent synchrony among a specific subpopulation of input neurons will have no more effect postsynaptically than the random collections of synchronous inputs that arise stochastically in the high-input regime. Synchronous spikes do not represent anything extraordinary.

\section{Concluding remarks}

Together, these principles inform a view of information coding by cortical neurons that draws diverse physiological and anatomical data into a coherent framework. In the high-input regime, neurons attain their dynamic range of response by balancing excitation with inhibition. As a consequence, they spike with great irregularity and lack the capacity to transmit information in patterns of spikes. The spike output for a single neuron therefore resembles a stochastic point process, and the rate term, $\lambda(t)$, is the currency of information transfer from neuron to neuron. Because the spikes from any one neuron arrive with such irregularity, however, $\lambda(t)$ must be represented in a population of neurons to gain signal/noise and, more importantly, speed. Neurons in such a signaling population must share common input, resulting in a certain amount of common noise that ultimately limits the fidelity of signal transmission. Within this limit, rate changes can be signaled in approximately one ISI using pools of $\sim 100$ neurons, but little or no improvement is gained with larger pools.

Finally, quantitative comparison of known noise sources and experimentally observed response variance suggests that a single neuron is unable to compute quantities much more complicated than a weighted average of the spikes arriving at its dendritic field. This implies that interesting neural response properties do not arise from sophisticated computation within single neurons but rather reflect the anatomical convergence of novel combinations of inputs to the ensemble (the column) from external sources. This insight is consistent with the basic computational principles incorporated in neural network models. From this point of view, the basic function of the column is to amplify these convergent inputs while controlling the gain of single neurons so that in any interspike interval sufficient spikes are available to represent appropriate quantities for subsequent computation. Several investigators have emphasized the importance of cortical amplification and gain control (Douglas and Martin, 1991; Heeger, 1992b; Douglas et al., 1995; Stratford et al., 1996).

Ideas about synaptic integration and ideas about neural computation tend to use different languages - the first involving conductances, membrane properties and spikes and the second having reference primarily to formal mathematical operations. Our analysis attempts to form a framework for unifying the view of 
the neuron as an integrator of synaptic input with the problem of what and how a neuron computes.

\section{APPENDIX 1}

Here we describe a simple conductance-based model with properties similar to the random walk counting model described in the text. Instead of counting to a threshold, we have modeled synaptic inputs as brief changes in $\mathrm{Na}^{+}$(excitatory inputs) or $\mathrm{Cl}^{-}$(inhibitory inputs) conductance, and solved for the membrane voltage. The membrane voltage, $V(t)$, is described by the differential equation:

$$
C_{\mathrm{m}} \frac{d V}{d t}=-\left(I_{\mathrm{Ex}}+I_{\mathrm{In}}+I_{\mathrm{K}}+I_{\text {leak }}\right)
$$

where $C_{\mathrm{m}}$ is the membrane capacitance, and $I$ represents the transmembrane current attributable to excitatory synaptic input $\left(I_{\mathrm{Ex}}\right)$, inhibitory input $\left(I_{\mathrm{In}}\right)$, a spike-triggered potassium current $\left(I_{\mathrm{K}}\right)$, and a leak current $\left(I_{\text {leak }}\right)$. The model contains just one compartment (i.e., no variation in $V$ as a function of location).

The synaptic currents were computed by multiplying a timevarying conductance by the driving force:

$$
\begin{gathered}
I_{\mathrm{Ex}}=g_{\mathrm{Ex}}(t)\left(V-E_{\mathrm{Na}}\right) ; \\
I_{\mathrm{In}}=g_{\mathrm{In}}(t)\left(V-E_{\mathrm{Cl}}\right) .
\end{gathered}
$$

The neuron receives 300 excitatory inputs and 300 inhibitory inputs. Each excitatory synaptic conductance was modeled by a simple exponential decay:

$$
g_{\text {Ex }}(t)=g_{\text {E_peak }} \exp \left(-t / \tau_{\text {Ex }}\right),
$$

with $g_{\text {E_peak }}=6 \mathrm{nS}$, and $\tau_{\mathrm{Ex}}=2 \mathrm{msec}$. The peak conductance was set so that 15 synchronous inputs would elicit a spike from rest $(-70 \mathrm{mV})$, as illustrated in Fig. 14B. Spike threshold was -55 $\mathrm{mV}$, which implies that the average EPSP was just $>1 \mathrm{mV}$ when the membrane was at rest. The inhibitory conductances were modeled similarly, but they were larger and longer-lasting ( $g_{\text {I_peak }}$ $\left.=11.25 \mathrm{nS} ; \tau_{\text {In }}=4 \mathrm{msec}\right)$. These parameters were chosen to balance the excitation, thereby yielding an output spike rate equal to the spike rates of the inputs (see below). An IPSP had no impact on the resting membrane, because $E_{\mathrm{Cl}}=V_{\text {rest }}=-70 \mathrm{mV}$. In addition to the synaptic conductance, we modeled a leak conductance, $I_{\text {leak }}=g_{\text {leak }}\left(V-V_{\text {rest }}\right)$, which was not time-varying. The leak conductance and membrane capacitance were chosen to achieve a $40 \mathrm{M} \Omega$ input resistance and $20 \mathrm{msec}$ time constant at rest $\left(g_{\text {leak }}=25 \mathrm{nS}\right)$.

Spikes were modeled as brief voltage transients, which we simply added to the membrane voltage: $\Delta V_{\mathrm{m}}=55 e^{-2 \mathrm{t}} \mathrm{mV}$ (i.e., a decay time constant of $0.5 \mathrm{msec}$ ). This model does not incorporate a voltage reset, but each spike triggered a $\mathrm{K}$ conductance (peak conductance, $0.15 \mu \mathrm{S} ; E_{\mathrm{K}}=-90 \mathrm{mV} ; \tau_{\mathrm{K}}=40 \mathrm{msec}$ ), leading to the spike frequency adaptation shown in Figure $14 A$. The $\mathrm{K}$ current, leak conductance and membrane capacitance produce the $f-I$ curve shown (Fig. $14 C$ ), which plots the steadystate firing rate as a function of current step amplitude. The model is similar to one described by Troyer and Miller (1996) except for the large number of synaptic inputs and absence of any reset voltage.

We stimulated the model with 300 excitatory inputs, each modeled as independent Poisson spike trains with an average rate of $80 \mathrm{spikes} / \mathrm{sec}$. The inhibitory inputs were of the same number and rate. We adjusted the peak inhibitory conductance so that the model produced an output of 78.9 spikes/sec. A half-second sample of the model neuron response is shown in Figure 14D. As with our counting model, the cost of a reasonable firing rate is an irregular ISI. This is apparent in the membrane voltage sample and in the distribution of ISIs (Fig. 14E) obtained from a $10 \mathrm{sec}$ simulation. The $C_{\mathrm{V}_{\mathrm{ISI}}}$ was 0.77 for this simulation, which is near the upper bound for a Poisson process with a refractory dead zone (Troyer and Miller, 1996).

To achieve a firing rate that approximates an average input, the peak inhibitory conductance was set to $11.25 \mathrm{nS}\left(\tau_{\mathrm{In}}=4\right.$ $\mathrm{msec}$ ). This led to a very large conductance, as shown in Figure $14 F$. With 300 excitatory and inhibitory inputs, the model incorporated a total synaptic conductance of $>600 \mathrm{nS}$, which is $\sim 25$ times the conductance at rest. Interestingly, this massive conductance change does not produce a large net current; only 2-4 nA is sufficient to produce the simulated spike trains in Figure $14 D$. In contrast to the large conductances incorporated in our model, Borg-Graham et al. (1996) measured a 200$300 \%$ change in conductance using whole-cell patch recordings of cortical neurons in vivo during periods of modest discharge (we know of no other comparable measurements of synaptically activated neurons under the high-input conditions that interest us). We suspect that the discrepancy results both from an underestimate of the actual conductance by Borg-Graham et al. (1996) and to an overestimate in our model. BorgGraham et al. (1996) estimated the conductance by measuring current-voltage relationships during voltage clamp of the cell soma. To the extent that the synapses are electrotonically distant from the soma, however, these measurements will inevitably underestimate synaptic conductance changes. On the other hand, our single-compartment model probably overestimates the total conductance. In a more realistic model, which includes the dendrites, it is likely that less inhibitory conductance would be required to balance a given amount of excitatory input [e.g., if excitatory synapses are further from the soma or if inhibitory synapses were to interfere with dendritic amplification (Cook and Johnston, 1997; Hoffman et al., 1997; Schwindt and Crill, 1997b)]. We therefore suspect that the actual state of affairs is intermediate between that estimated by Borg-Graham et al. (1996) and that incorporated in our model. Measurements of the dendritic conductance during vigorous sensory stimulation will be necessary to resolve this discrepancy fully.

There is one aspect of the simulation that we regard as deceptive. The membrane voltage appears to hover near spike threshold and to cross occasionally at random intervals, resembling the coincidence detector depicted in Figure 2, $D$ and $E$. In light of the large conductance, it might be argued that the membrane time constant is effectively shortened from 20 to $<1 \mathrm{msec}$ (Koch et al., 1995), and that therefore the model neuron only spikes when it senses rare coincidences of excitatory input within a time frame of $\sim 1 \mathrm{msec}$. Although the effective integration time is indeed short, spikes are not the result of an occasional volley of excitation. As shown in Figure $14 F$, the events preceding a spike include a pause in the inhibition as well as a rise in excitation. In this simulation the neuron was affected by synaptic input throughout much of the ISI (mean ISI was $14 \mathrm{msec}$ ). Moreover, the increased excitation immediately preceding a spike hardly constitutes a rare coincidence. The change in excitatory conductance (Fig. 14F, solid line) was just $20 \mathrm{nS}$, on average, consistent with just three or four extra synaptic inputs over the background. In the simulation depicted 

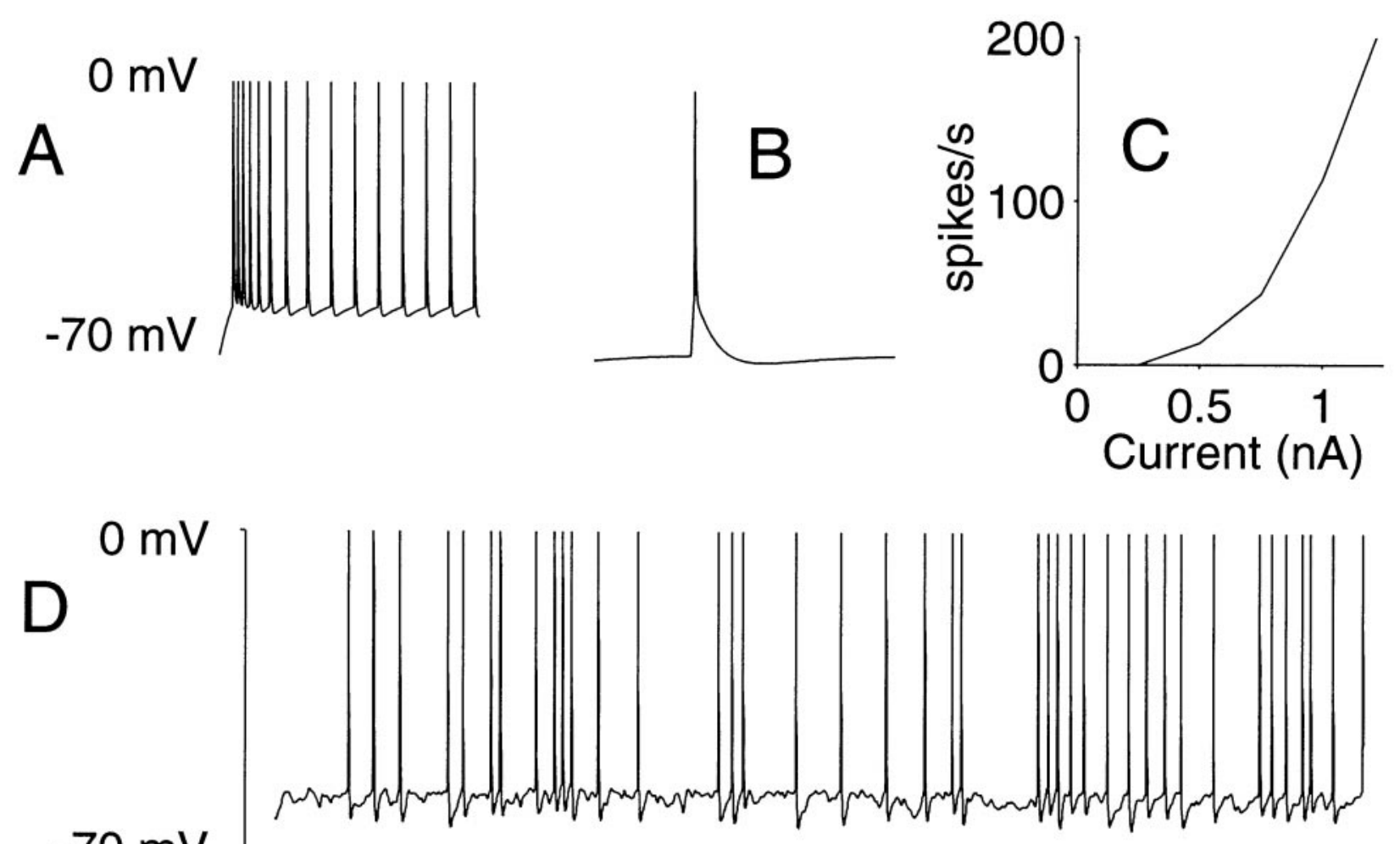

$-70 \mathrm{mV}$

\section{$0 \mathrm{~ms}$}

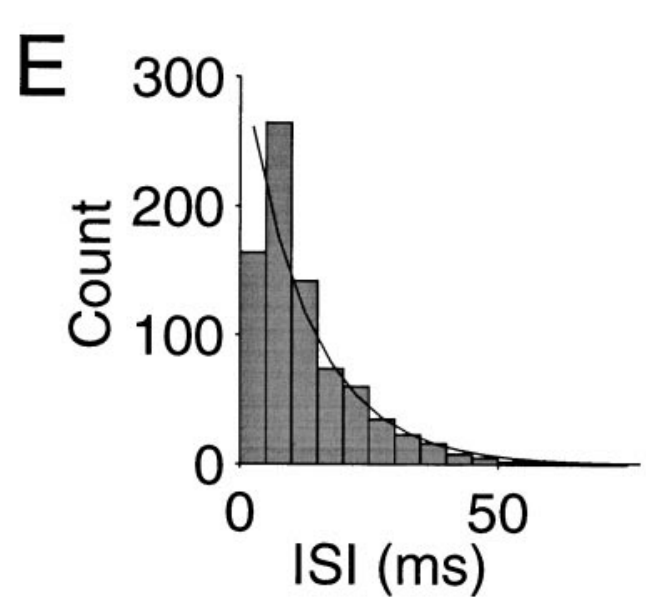

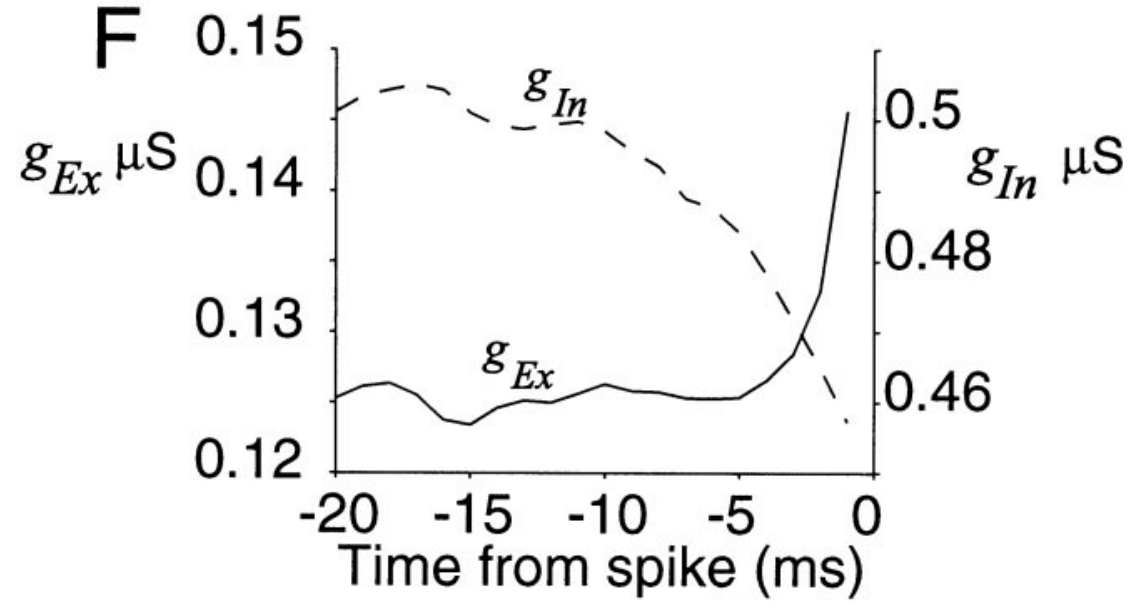

Figure 14. A single-compartment model using brief synaptic conductance changes instead of voltage steps. The model behaves like the simpler random walk counting model. The resting potential is $-70 \mathrm{mV}, \tau_{\mathrm{m}}=20 \mathrm{msec}$, and spike threshold is $-55 \mathrm{mV}$. $A$, Response to $0.75 \mathrm{nA}$ current step. The trace lasts $250 \mathrm{msec}$. $B$, Fifteen synchronous excitatory synaptic inputs depolarize the neuron sufficiently to trigger a spike. $C$, Response to simulated current steps of varying size. The $f-I$ plot depicts the steady-state firing rate as a function of injected current. $D$, Response to 300 excitatory and 300 inhibitory inputs. Each input neuron spikes at an average of 80 spikes/sec. The size of the inhibitory conductance was adjusted to produce an average spike rate similar to the input rate. Notice that the membrane voltage hovers near spike threshold. $E$, ISI distribution from the simulated response, which includes the trace in $C$. Solid line is an exponential fit to the distribution. F, Average synaptic conductance preceding a spike. The spike-triggered average excitatory conductance is shown by the solid curve (left ordinate scale); the inhibitory conductance is shown by the dashed curve (right ordinate scale). Although the effective membrane time constant is $\sim 0.5 \mathrm{msec}$, the synaptic activity affects the neuron for $5-10$ msec preceding a spike.

here, such excesses occur in 1 of 4 msec. In other words, the putative coincidences are ubiquitous. In general, spikes are not the consequence of particular patterns of excitatory input, because the sequence of inhibitory inputs also affects the time of the postsynaptic spike. Inhibition does not merely shorten the integration time for excitation. Thus, unlike the coincidence detector portrayed in Figure 2, $D$ and $E$, the present model would require a fine orchestration of excitatory and inhibitory synaptic input to produce reliably timed spikes. This insight is likely to hold for other biophysical implementations of balanced excitation-inhibition, as long as the balancing inhibition comes from spiking neurons. 
The conductance-based model described here embodies the same constraints as our simpler counting model, and it achieves a highly irregular ISI. It is just one example of a biophysical implementation of the random walk to a barrier idea that solves the problem faced by neurons that receive a surfeit of excitatory input. Although the conduction-based model may be more biophysically plausible than the counting model pursued in the main text, it remains highly simplified and sacrifices much of the conceptual simplicity inherent in counting to a barrier. It would be useful to determine whether a more realistic model using active dendritic conductances (e.g., Cook and Johnston, 1997) would behave similarly.

\section{APPENDIX 2}

Here we derive the standard error of the mean spike count from $m$ weakly correlated neurons over a duration lasting one average ISI. Throughout the paper we refer to a computation of the average input spike rate, in which the expected output spike rate is the same, on average, as any one of its active inputs. Equation 7 expresses the expected spike rate as a function of the $m$ input spike counts transmitted in an epoch, $T$. The expected spike count is:

$$
\langle\lambda T\rangle=\frac{1}{m} \sum_{i=1}^{m} n_{\mathrm{i}}(T)
$$

Each of the $m$ inputs is a random quantity with variance denoted $\operatorname{var}\left[n_{\mathrm{i}}(T)\right]$. If the $m$ input counts are independent, then the variance of the mean is just the average variance of one input, divided by $m$ :

$$
\operatorname{Var}[\langle\lambda T\rangle]=\frac{1}{m}\left\langle\operatorname{Var}\left[n_{\mathrm{i}}(T)\right]\right\rangle_{\mathrm{i}}=\frac{1}{m^{2}} \sum_{i=1}^{m} \operatorname{Var}\left[n_{\mathrm{i}}(T)\right],
$$

where $\langle\cdots\rangle_{\mathrm{i}}$ denotes the average across all values of $i$. If the $m$ inputs are weakly correlated, then the variance of the mean is derived from the sum of the covariance among the $m$ inputs:

$$
\begin{aligned}
\operatorname{Var}[\langle\lambda T\rangle] & =\frac{1}{m^{2}} \sum_{i=1}^{\mathrm{m}} \sum_{j=1}^{\mathrm{m}} \operatorname{Cov}\left[n_{\mathrm{i}}, n_{\mathrm{j}}\right] \\
& =\frac{1}{m^{2}}\left(\sum_{i=1}^{\mathrm{m}} \operatorname{Var}\left[n_{\mathrm{i}}(T)\right]+\sum_{i=1}^{\mathrm{m}} \sum_{j \neq \mathrm{i}}^{\mathrm{m}} r_{\mathrm{ij}} \sqrt{\operatorname{Var}\left[n_{\mathrm{i}}(T)\right] \operatorname{Var}\left[n_{\mathrm{j}}(T)\right]}\right),
\end{aligned}
$$

where $r_{\mathrm{ij}}$ is the correlation coefficient between the $i^{\text {th }}$ and $j^{\text {th }}$ input. This is just the sum of the elements forming the $m$-by- $m$ covariance matrix. If all $m$ inputs are identically distributed, then we can eliminate the subscripts on the terms for variance and substitute the mean correlation coefficient, $\bar{r}$, for $r_{\mathrm{ij}}$ :

$$
\begin{aligned}
\operatorname{Var}[\langle\lambda T\rangle] & =\frac{1}{m^{2}}(m \operatorname{Var}[n(T)]+m(m-1) \bar{r} \operatorname{Var}[n(T)]) \\
& \approx \bar{r} \operatorname{Var}[n(T)], \quad \text { for large } m
\end{aligned}
$$

If the $m$ inputs do not share the same mean and variance, as is often the case, then a useful approximation is to substitute the average variance:

$$
\operatorname{Var}[\langle\lambda T\rangle] \approx \bar{r}\left\langle\operatorname{Var}\left[n_{\mathrm{i}}(T)\right]\right\rangle_{\mathrm{i}}, \quad \text { for large } m
$$

In practice, $m$ need only be $\geq 100$ for the approximations in Equations A2.4 and A2.5 to hold.

For the special case in which $n_{\mathrm{i}}(T)$ is the count of events in a Poisson point process, and the duration, $T_{\text {ISI }}=\lambda^{-1}$, is the expected ISI, we have:

$$
\operatorname{Var}\left[n_{\mathrm{i}}\left(T_{\text {ISI }}\right)\right]=\left\langle n_{\mathrm{i}}\left(T_{\text {ISI }}\right)\right\rangle=1 .
$$

Substituting into Equation A2.4 yields:

$$
\operatorname{Var}\left[\left\langle\lambda T_{\mathrm{ISI}}\right\rangle\right]=\frac{1+m \bar{r}-\bar{r}}{m},
$$

from which follows the expression in Equation 3 and the curves in Figure 10.

\section{REFERENCES}

Abbott LF (1994) Decoding neuronal firing and modelling neural networks. Q Rev Biophys 27:291-331.

Abbott LF, Varela JA, Sen K, Nelson SB (1997) Synaptic depression and cortical gain control. Science 275:220-224.

Abeles M (1982) Role of cortical neuron: integrator or coincidence detector? Isr J Med Sci 18:83-92.

Abeles M (1991) Corticonics. Neural circuits of the cerebral cortex. Cambridge, UK: Cambridge UP.

Abeles M, Bergman H, Margalit E, Vaadia E (1993) Spatiotemporal firing patterns in the frontal cortex of behaving monkeys. J Neurophysiol 70:1629-1638.

Adelson EH, Bergen JR (1985) Spatio-temporal energy models for the perception of motion. J Opt Soc Am A 2:284-299.

Aertsen A, Diesmann M, Gewaltig MO (1996) Propagation of synchronous spiking activity in feedforward neural networks. J Physiol (Paris) 90:243-247.

Albright TD (1993) Cortical processing of visual motion. In: Visual motion and its role in the stabilization of gaze (Miles FA, Wallman J, eds), pp 177-201. New York: Elsevier.

Amir Y, Harel M, Malach R (1993) Cortical hierarchy reflected in the organization of intrinsic connections in macaque monkey visual cortex. J Comp Neurol 334:19-46.

Amitai Y, Friedman A, Connors B, Gutnick M (1993) Regenerative activity in apical dendrites of pyramidal cells in neocortex. Cereb Cortex 3:26-38.

Arieli A, Sterkin A, Grinvald A, Aertsen A (1996) Dynamics of ongoing activity: explanation of the large variability in evoked cortical responses. Science 273:1868-1871.

Bair W, Koch C (1996) Temporal precision of spike trains in extrastriate cortex. Neural Comp 8:1185-1202.

Bair W, O'Keefe LP (1998) The influence of fixational eye movements on the response of neurons in area MT of the macaque. Vis Neurosci, in press.

Beaulieu C, Kisvarday Z, Somogyi P, Cynader M, Cowey A (1992) Quantitative distribution of GABA-immunopositive and -immuno negative neurons and synapses in the monkey striate cortex (area 17). Cereb Cortex 2:295-309.

Bernander Ö, Koch C, Douglas RJ (1994) Amplification and linearization of distal synaptic input to cortical pyramidal cells. J Neurophysiol 72:2743-2752.

Borg-Graham L, Monier C, Fregnac Y (1996) Voltage-clamp measurement of visually-evoked conductances with whole-cell patch recordings in primary visual cortex. J Physiol (Paris) 90:185-188.

Bosking WH, Zhang Y, Schofield B, Fitzpatrick D (1997) Orientation selectivity and the arrangement of horizontal connections in tree shrew striate cortex. J Neurosci 17:2112-2127.

Bradley A, Skottun BC, Ohzawa I, Sclar G, Freeman RD (1987) Visual orientation and spatial frequency discrimination: a comparison of single cells and behavior. J Neurophysiol 57:755-772.

Braitenber V, Schüz A (1991) Anatomy of the cortex. Statistics and geometry. Berlin: Springer. 
Britten KH, Shadlen MN, Newsome WT, Movshon JA (1992) The analysis of visual motion: a comparison of neuronal and psychophysical performance. J Neurosci 12:4745-4765.

Britten KH, Shadlen MN, Newsome WT, Movshon JA (1993) Responses of neurons in macaque MT to stochastic motion signals. Vis Neurosci 10:1157-1169.

Calvin WH, Stevens CF (1968) Synaptic noise and other sources of randomness in motoneuron interspike intervals. J Neurophysiol 31:574-587.

Carandini M, Ferster D (1997) A tonic hyperpolarization underlying contrast adaptation in cat visual cortex. Science 276:949-952.

Carvell GE, Simons DJ (1988) Membrane potential changes in rat SmI cortical neurons evoked by controlled stimulation of mystacial vibrissae. Brain Res 448:186-191.

Cauller L, Connors B (1994) Synaptic physiology of horizontal afferents to layer I in slices of rat SI neocortex. J Neurosci 14:751-762.

Celebrini S, Newsome WT (1994) Neuronal and psychophysical sensitivity to motion signals in extrastriate area MST of the macaque monkey. J Neurosci 14:4109-4124.

Cook EP, Johnston D (1997) Active dendrites reduce locationdependent variability of synaptic input trains. J Neurophysiol 78:2116-2128.

Das A, Gilbert CD (1995a) Long-range horizontal connections and their role in cortical reorganization revealed by optical recording of cat primary visual cortex. Nature 375:780-784.

Das A, Gilbert CD (1995b) Receptive field expansion in adult visual cortex is linked to dynamic changes in strength of cortical connections. J Neurophysiol 74:779-792.

Dean AF (1981) The variability of discharge of simple cells in cat striate cortex. Exp Brain Res 44:437-440.

DeFelipe J, Jones E (1985) Vertical organization of $\gamma$-aminobutyric acid-accumulating intrinsic neuronal systems in monkey cerebral cortex. J Neurosci 5:3246-3260.

Douglas RH, Martin KAC (1991) A functional microcircuit for cat visual cortex. J Physiol (Lond) 440:735-769.

Douglas RJ, Martin KAC, Witteridge D (1991) An intracellular analysis of the visual responses of neurones in cat visual cortex. J Physiol (Lond) 440:659-696.

Douglas RJ, Koch C, Mahowald M, Martin KAC, Suarez HH (1995) Recurrent excitation in neocortical circuits. Science 269:981-985.

Engel A, Konig P, Kreiter A, Schillen T, Singer W (1992) Temporal coding in the visual cortex: new vistas on integration in the nervous system. Trends Neurosci 15:218-226.

Ferster D (1986) Orientation selectivity of synaptic potentials in neurons of cat primary visual cortex. J Neurosci 6:1284-1301.

Ferster D, Chung S, Wheat H (1996) Orientation selectivity of thalamic input to simple cells of cat visual cortex. Nature 380:249-252.

Fetz E, Toyama K, Smith W (1991) Synaptic interactions between cortical neurons. In: Cerebral cortex, Vol 9 (Peters A, Jones EG, eds), pp 1-47. New York: Plenum.

Funke K, Worgotter F (1995) Temporal structure in the light response of relay cells in the dorsal lateral geniculate nucleus of the cat. J Physiol (Lond) 485:715-737.

Gawne TJ, Richmond BJ (1993) How independent are the messages carried by adjacent inferior temporal cortical neurons? J Neurosci 13:2758-2771.

Geisler WS, Albrecht DG (1997) Visual cortex neurons in monkeys and cats: detection, discrimination and identification. Vis Neurosci 14:987-920.

Gerstein G, Mandelbrot B (1964) Random walk models for the spike activity of a single neuron. Biophys J 4:41-68.

Gilbert CD, Wiesel TN (1989) Columnar specificity of intrinsic horizontal and corticocortical connections in cat visual cortex. J Neurosci 9:2432-2442.

Gray CM, McCormick DA (1996) Chattering cells: superficial pyramidal neurons contributing to the generation of synchronous oscillations in the visual cortex. Science 274:339-340.

Grinvald A, Lieke EE, Frostig RD, Hildesheim R (1994) Cortical pointspread function and long-range lateral interactions revealed by realtime optical imaging of macaque monkey primary visual cortex. J Neurosci 14:2545-2568.

Gur M, Beylin A, Snodderly DM (1997) Response variability of neurons in primary visual cortex (V1) of alert monkeys. J Neurosci 17:2914-2920.

Hawken MJ, Parker AJ (1990) Detection and discrimination mecha- nisms in the striate cortex of the old-world monkey. In: Vision: coding and efficiency (Blakemore C, ed), pp 103-116. Cambridge, UK: Cambridge UP.

Heeger DJ (1992a) Half-squaring in responses of cat striate cells. Vis Neurosci 9:427-443.

Heeger DJ (1992b) Normalization of cell responses in cat striate cortex. Vis Neurosci 9:181-198.

Heeger DJ, Simoncelli EP, Movshon JA (1996) Computational models of cortical visual processing. Proc Natl Acad Sci USA 93:623-627.

Hellwig B, Schüz A, Aertsen A (1994) Synapses on axon collaterals of pyramidal cells are spaced at random intervals: a Golgi study in the mouse cerebral cortex. Biol Cybern 71:1-12.

Hoffman DA, Magee JC, Colbert CM, Johnston D (1997) $\mathrm{K}^{+}$channel regulation of signal propagation in dendrites of hippocampal pyramidal neurons. Nature 387:869-875.

Hoffmann K-P, Stone J, Sherman SM (1972) Relay of receptive-field properties in dorsal lateral geniculate nucleus of the cat. J Neurophysiol 35:518-531.

Hubel DH, Wiesel TN (1961) Integrative action in the cat's lateral geniculate body. J Physiol (Lond) 155:385-398.

Johnson KO (1980) Sensory discrimination: decision process. J Neurophysiol 43:1771-1792.

Johnston D, Magee JC, Colbert CM, Christie BR (1996) Active properties of neuronal dendrites. Annu Rev Neurosci 19:165-186.

Kaplan E, Shapley RM (1984) The origin of the S (slow) potential in the mammalian lateral geniculate nucleus. Exp Brain Res 55:111-116.

Karlin S, Taylor HM (1975) A first course in stochastic processes. Boston: Academic.

Kenyon GT, Fetz EE, Puff RD (1990) Effects of firing synchrony on signal propagation in layered networks. In: Advances in neural information processing systems 2 (Fouretzky DD, ed), pp 141-148. San Mateo, CA: Kaufmann.

Kisvarday Z, Beaulieu C, Eysel U (1993) Network of GABAergic large basket cells in cat visual cortex (area 18): implication for lateral disinhibition. J Comp Neurol 327:398-415.

Knight BW (1972) Dynamics of encoding in a population of neurons. J Gen Physiol 59:734-766.

Koch C, Poggio T, Torre V (1983) Nonlinear interactions in a dendritic tree: localization, timing, and role in information processing. Proc Natl Acad Sci USA 80:2799-2802.

Koch C, Bernander O, Douglas RJ (1995) Do neurons have a voltage or a current threshold for action potential initiation? J Comput Neurosci 2:63-82.

Konig P, Engel AK, Singer W (1996) Integrator of coincidence detector? The role of the cortical neuron revisited. Trends Neurosci 19:130-137.

Lansky P, Lanska V (1987) Diffusion approximation of the neuronal model with synaptic reversal potentials. Biol Cybern 56:19-26.

Lee D, Port NL, Kruse W, Georgopoulos AP (1998) Variability and correlated noise in the discharge of neurons in motor and parietal areas of the primate cortex. J Neurosci 18:1161-1170.

Lestienne R (1996) Determination of the precision of spike timing in the visual cortex of anaesthetised cats. Biol Cybern 74:55-61.

Lumer ED, Edelman GM, Tononi G (1997) Neural dynamics in a model of the thalamocortical system. II. The role of neural synchrony tested through perturbations of spike timing. Cereb Cortex 7:228-236.

Lund J, Yoshioka T, Levitt J (1993) Comparison of intrinsic connectivity in different areas of macaque monkey cerebral cortex. Cereb Cortex 3:148-162.

Mainen ZF, Sejnowski TJ (1995) Reliability of spike timing in neocortical neurons. Science 268:1503-1506.

Markram H, Tsodyks M (1996) Redistribution of synaptic efficacy between neocortical pyramidal neurons. Nature 382:807-810.

Mason A, Nicoll A, Stratford K (1991) Synaptic transmission between individual pyramidal neurons of the rat visual cortex in vitro. J Neurosci 11:72-84.

Matsumura M, Chen D-F, Sawaguchi T, Kubota K, Fetz EE (1996) Synaptic interactions between primate precentral cortex neurons revealed by spike-triggered averaging of intracellular membrane potentials in vivo. J Neurosci 16:7757-7767.

McCasland JS, Hibbard LS (1997) GABAergic neurons in barrel cortex show strong, whisker-dependent metabolic activation during normal behavior. J Neurosci 17:5509-5527. 
McCormick D, Connors B, Lighthall J, Prince D (1985) Comparative electrophysiology of pyramidal and sparsely spiny stellate neurons in the neocortex. J Neurophysiol 54:782-806.

McIlwain J (1990) Large receptive fields and spatial transformations in the visual system. In: Neurocomputing 2, Directions for research. (Anderson JA, Pellionisz A, Rosenfeld E, eds), pp 239-253. Cambridge, MA: MIT.

Meister M (1996) Multineuronal codes in retinal signaling. Proc Natl Acad Sci USA 93:609-614.

Mel BW (1993) Synaptic integration in an excitable dendritic tree. J Neurophysiol 70:1086-1101.

Melssen WJ, Epping WJM (1987) Detection and estimation of neural connectivity based on crosscorrelation analysis. Biol Cybern $57: 403-414$.

Mood AM, Grabill FA, Boes DC (1963) Introduction to the theory of statistics. New York: McGraw-Hill.

Moore GP, Segundo JP, Perkel DH, Levitan H (1970) Statistical signs of synaptic interaction in neurons. Biophys J 10:876-900.

Mountcastle VB (1957) Modality and topographic properties of single neurons of cat's somatic sensory cortex. J Neurophysiol 20:408-434.

Mountcastle VB (1978) An organizing principle for cerebral function: the unit module and the distributed system. In: The mindful brain (Edelman GM, Mountcastle VB, eds), pp 7-50. Cambridge, MA: MIT.

Movshon J, Newsome W (1996) Visual response properties of striate cortical neurons projecting to area MT in macaque monkeys. J Neurosci 16:7733-7741.

Murthy VN, Fetz EE (1994) Effects of input synchrony on the firing rate of a three-conductance cortical neuron model. Neural Comput 6:1111-1126.

Nelson SB, Toth LJ, Sheth BR, Sur M (1995) Orientation selectivity of cortical neurons persists during intracellular blockade of inhibition. Science 265:774-777.

Nowak LG, Munk MHJ, Nelson JI, James AC, Bullier J (1995) Structural basis of cortical synchronization. I. Three types of interhemispheric coupling. J Neurophysiol 74:2379-2400.

Nowak LG, Sanchez-Vives MV, McCormick DA (1997) Influence of low and high frequency inputs on spike timing in visual cortical neurons. Cereb Cortex 7:487-501.

Oertel D (1983) Synaptic responses and electrical properties of cells in brain slices of the mouse anteroventral cochlear nucleus. J Neurosci 3:2043-2053.

Otmakhov N, Shirke AM, Malinow R (1993) Measuring the impact of probabilistic transmission on neuronal output. Neuron 10:1101-1111.

Parker A, Hawken M (1985) Capabilities of monkey cortical cells in spatial-resolution tasks. J Opt Soc Am A 2:1101-1114.

Peters A (1987) Synaptic specificity in the cerebral cortex. In: Synaptic function (Edelman G, Gall W, Cowan W, eds), pp 373-397. New York: Wiley.

Peters A, Sethares C (1991) Organization of pyramidal neurons in area 17 of monkey visual cortex. J Comp Neurol 306:1-23.

Pouget A, Zhang K, Deneve S, Latham PE (1998) Statistically efficient estimation using population code. Neural Comput 10:373-401.

Reid RC, Alonso JM (1995) Specificity of monosynaptic connections from thalamus to visual cortex. Nature 378:281-284.

Reyes AD, Fetz EE (1993) Two modes of interspike interval shortening by brief transient depolarizations in cat neocortical neurons. J Neurophysiol 69:1661-1672.

Ricciardi L, Sacerdote L (1979) The Ornstein-Uhlenbeck process as a model for neuronal activity. I. Mean and variance of the firing time. Biol Cybern 35:1-9.

Rieke F, Warland D, de Ruyter van Steveninck RR, Bialek W (1997) Spikes: exploring the neural code. Cambridge, MA: MIT.

Schiller PH, Finlay B, Volman S (1976) Short-term response variability of monkey striate neurons. Brain Res 105:347-349.

Schwindt PC, Crill WE (1995) Amplification of synaptic current by persistent sodium conductance in apical dendrite of neocortical neurons. J Neurophysiol 74:2220-2224.

Schwindt PC, Crill WE (1997a) Local and propagated dendritic action potentials evoked by glutamate iontophoresis on rat neocortical pyramidal neurons. J Neurophysiol 77:2466-2483.

Schwindt PC, Crill WE (1997b) Modification of current transmitted from apical dendrite to soma by blockade of voltage- and $\mathrm{Ca}^{2+}$. dependent conductances in rat neocortical pyramidal neurons. J Neurophysiol 78:187-199.
Scobey RP, Gabor AJ (1989) Orientation discrimination sensitivity of single units in cat primary visual cortex. Exp Brain Res 77:398-406.

Segev I (1992) Single neurone models: oversimple, complex and reduced. Trends Neurosci 15:414-421.

Seung HS, Sompolinsy H (1993) Simple models for reading neuronal population codes. Proc Natl Acad Sci USA 90:10749-10753.

Shadlen MN, Newsome WT (1994) Noise, neural codes and cortical organization. Curr Opin Neurobiol 4:569-579.

Shadlen MN, Britten KH, Newsome WT, Movshon JA (1996) A computational analysis of the relationship between neuronal and behavioral responses to visual motion. J Neurosci 16:1486-1510.

Shepherd GM, Brayton RK (1987) Logic operations are properties of computer-simulated interactions between excitable dendritic spines. Neuroscience 21:151-165.

Simoncelli EP, Heeger DJ (1998) A velocity population coding model of MT neurons. Vision Res 38:743-761

Singer W (1994) Putative functions of temporal correlations in neocortical processing. In: Large-scale neuronal theories of the brain (Koch C, Davis JL, eds), pp 201-237. Cambridge, MA: MIT.

Smith WL (1959) On the cumulants of renewal processes. Biometrika 46:1-29.

Snowden RJ, Treue S, Andersen RA (1992) The response of neurons in areas V1 and MT of the alert rhesus monkey to moving random dot patterns. Exp Brain Res 88:389-400.

Softky W (1994) Sub-millisecond coincidence detection in active dendrite trees. Neuroscience 58:13-41.

Softky WR, Koch C (1993) The highly irregular firing of cortical cells is inconsistent with temporal integration of random EPSPs. J Neurosci 13:334-350.

Somers DC, Nelson SB, Sur M (1995) An emergent model of orientation selectivity in cat visual cortical simple cells. J Neurosci 15:5448-5465.

Somogyi P (1989) Synaptic organization of GABAergic neurons and GABA-A receptors in the lateral geniculate nucleus and visual cortex. In: Neural mechanisms of visual perception. Proceedings of the retina research foundation, Vol 2. (Lam DM-K, Gilbert CD, eds), pp 35-62. The Woodlands, TX: Portfolio Publishing.

Somogyi P, Cowey A, Kisvarday Z, Freund T, Szentagothai J (1983a) Retrograde transport of $\gamma$-amino $\left[{ }^{3} \mathrm{H}\right]$ butyric acid reveals specific interlaminar connections in the striate cortex of monkey. Proc Natl Acad Sci USA 80 .

Somogyi P, Kisvarday ZF, Martin KAC, Whitteridge D (1983b) Synaptic connections of morphologically identified and physiologically characterized large basket cells in the striate cortex of cat. Neuroscience $10: 261-294$

Stein R (1965) A theoretical analysis of neuronal variability. Biophys J 5:173-194.

Stratford KJ, Tarczy-Hornoch K, Martin KAC, Bannister NJ, Jack JJB (1996) Excitatory synaptic inputs to spiny stellate cells in cat visual cortex. Nature 382:258-261.

Stuart G, Sakmann B (1995) Amplification of EPSPs by axosomatic sodium channels in neocortical pyramidal neurons. Neuron 15:1065-1076.

Tanaka K (1983) Cross-correlation analysis of geniculostriate neuronal relationships in cats. J Neurophysiol 49:1303-1319.

Teich MC, Heneghan C, Lowen SB, Ozaki T, Kaplan E (1997) Fractal character of the neural spike train in the visual system of the cat. J Opt Soc Am A 14:529-546.

Thomson A, West D (1993) Fluctuations in pyramid-pyramid excitatory postsynaptic potentials modified by presynaptic firing pattern and postsynaptic membrane potential using paired intracellular recordings in rat neocortex. Neuroscience 54:329-346.

Thomson A, Deuchars J, West D (1993a) Large, deep layer pyramidpyramid single axon EPSPs in slices of rat motor cortex display paired pulse and frequency-dependent depression, mediated presynaptically and self-facilitation, mediated postsynaptically. J Neurophysiol 70:2354-2369.

Thomson A, Deuchars J, West D (1993b) Single axon excitatory postsynaptic potentials in neocortical interneurons exhibit pronounce paired pulse facilitation. Neuroscience 54:347-360.

Thomson AM, West DC, Hahn J, Deuchars J (1996) Single axon IPSPs elicited in pyramidal cells by three classes of interneurones in slices of rat neocortex. J Physiol (Lond) 496:81-102.

Tolhurst DJ, Movshon JA, Dean AF (1983) The statistical reliability of signals in single neurons in cat and monkey visual cortex. Vision Res 23:775-785. 
Tomko G, Crapper D (1974) Neuronal variability: non-stationary responses to identical visual stimuli. Brain Res 79:405-418.

Troyer TW, Miller KD (1996) Physiological gain leads to hight ISI variability in a simple model of a cortical regular spiking cell. Neural Comput 9:971-983.

Ts'o DY, Gilbert CD, Wiesel TN (1986) Relationships between horizontal interactions and functional architecture in cat striate cortex as revealed by cross-correlation analysis. J Neurosci 6:1160-1170.

Tuckwell HC (1988) Introduction to theoretical neurobiology: Vol 2, Nonlinear and stochastic theories. Cambridge, UK: Cambridge UP.

van Kan PLE, Scobey RP, Gabor AJ (1985) Response covariance in cat visual cortex. Exp Brain Res 60:559-563. van Vreeswijk C, Sompolinsky H (1996) Chaos in neuronal networks with balanced excitatory and inhibitory activity. Science 274:1724-1726.

Vogels R, Spileers W, Orban GA (1989) The response variability of striate cortical neurons in the behaving monkey. Exp Brain Res 77:432-436.

Weliky M, Kandler K, Fitzpatrick D, Katz LC (1995) Patterns of excitation and inhibition evoked by horizontal connections in visual cortex share a common relationship to orientation columns. Neuron 15:541-552.

Zohary E, Shadlen MN, Newsome WT (1994) Correlated neuronal discharge rate and its implications for psychophysical performance. Nature 370:140-143. 